\title{
A Survey of Atomic Carbon [C i] in High-redshift Main-sequence Galaxies
}

\author{
Valentino, Francesco ; Magdis, Georgios E.; Daddi, Emanuele; Liu, Daizhong; Aravena, Manuel; \\ Bournaud, Frédéric; Cibinel, Anna; Cormier, Diane; Dickinson, Mark E.; Gao, Yu \\ Total number of authors: \\ 18
}

Published in:

Astrophysical Journal Supplement Series

Link to article, DOI:

10.3847/1538-4357/aaeb88

Publication date:

2018

Document Version

Publisher's PDF, also known as Version of record

Link back to DTU Orbit

Citation (APA):

Valentino, F., Magdis, G. E., Daddi, E., Liu, D., Aravena, M., Bournaud, F., Cibinel, A., Cormier, D., Dickinson, M. E., Gao, Y., Jin, S., Juneau, S., Kartaltepe, J., Lee, M-Y., Madden, S. C., Puglisi, A., Sanders, D., \& Silverman, J. (2018). A Survey of Atomic Carbon [C i] in High-redshift Main-sequence Galaxies. Astrophysical Journal Supplement Series, 869(1), [27]. https://doi.org/10.3847/1538-4357/aaeb88

\section{General rights}

Copyright and moral rights for the publications made accessible in the public portal are retained by the authors and/or other copyright owners and it is a condition of accessing publications that users recognise and abide by the legal requirements associated with these rights.

- Users may download and print one copy of any publication from the public portal for the purpose of private study or research.

- You may not further distribute the material or use it for any profit-making activity or commercial gain

- You may freely distribute the URL identifying the publication in the public portal 


\title{
A Survey of Atomic Carbon [CI] in High-redshift Main-sequence Galaxies
}

\author{
Francesco Valentino ${ }^{1,2}$ (1) , Georgios E. Magdis ${ }^{1,2,3}$ (1) Emanuele Daddi $^{4}\left(\mathbb{C}\right.$, Daizhong Liu ${ }^{5}$ (1), Manuel Aravena ${ }^{6}$ (1),

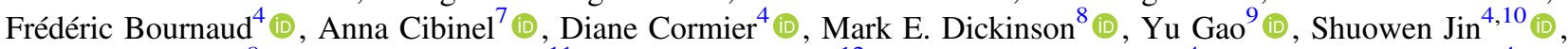 \\ Stéphanie Juneau ${ }^{8}$ (1), Jeyhan Kartaltepe ${ }^{11}$ (1) , Min-Young Lee ${ }^{12}$ (1) , Suzanne C. Madden ${ }^{4}$ (1), Annagrazia Puglisi ${ }^{4}$ (1), \\ David Sanders ${ }^{13}$ (1) and John Silverman ${ }^{14}$ (1) \\ ${ }^{1}$ Cosmic Dawn Center (DAWN), Niels Bohr Institute, University of Copenhagen, Juliane Maries Vej 30, DK-2100 Copenhagen Ø; DTU-Space, Technical University \\ of Denmark, Elektrovej 327, DK-2800 Kgs. Lyngby; francesco.valentino@nbi.ku.dk \\ ${ }^{2}$ Dark Cosmology Centre, Niels Bohr Institute, University of Copenhagen, Juliane Maries Vej 30, DK-2100 Copenhagen, Denmark \\ ${ }^{3}$ Institute for Astronomy, Astrophysics, Space Applications and Remote Sensing, National Observatory of Athens, GR-15236 Athens, Greece \\ ${ }^{4}$ Laboratoire AIM-Paris-Saclay, CEA/DSM-CNRS-Université Paris Diderot, Irfu/Service d'Astrophysique, CEA Saclay, Orme des Merisiers, F-91191 Gif sur \\ Yvette, France \\ ${ }^{5}$ Max Planck Institute for Astronomy, Königstuhl 17, D-69117 Heidelberg, Germany \\ ${ }^{6}$ Núcleo de Astronomía, Facultad de Ingeniería y Ciencias, Universidad Diego Portales, Av. Ejrcito 441, Santiago, Chile \\ ${ }^{7}$ Astronomy Centre, Department of Physics and Astronomy, University of Sussex, Brighton, BN1 9QH, UK \\ ${ }^{8}$ National Optical Astronomy Observatory, 950 North Cherry Avenue, Tucson, AZ 85719, USA \\ ${ }^{9}$ Purple Mountain Observatory/Key Laboratory for Radio Astronomy, Chinese Academy of Sciences, 6 YuanHua Road, Nanjing 210034, People's Republic of \\ China \\ ${ }^{10}$ School of Astronomy and Space Science, Nanjing University, Nanjing 210093, People's Republic of China \\ ${ }^{11}$ School of Physics and Astronomy, Rochester Institute of Technology, 84 Lomb Memorial Drive, Rochester, NY 14623, USA \\ ${ }^{12}$ Max-Planck-Institut für Radioastronomie, Auf dem Hegel 69, D-53121 Bonn, Germany \\ ${ }^{13}$ University of Hawaii, Institute for Astronomy, 2680 Woodlawn Drive, Honolulu, HI 96822, USA \\ ${ }^{14}$ Kavli Institute for the Physics and Mathematics of the Universe, Todai Institutes for Advanced Study, the University of Tokyo, Kashiwa, Japan 277-8583 (Kavli \\ IPMU, WPI) \\ Received 2018 July 12; revised 2018 October 15; accepted 2018 October 23; published 2018 December 7
}

\begin{abstract}
We present the first results of an Atacama Large Millimeter Array survey of the lower fine-structure line of atomic carbon $[\mathrm{CI}]\left({ }^{3} P_{1}-{ }^{3} P_{0}\right)$ in far-infrared-selected galaxies on the main sequence at $z \sim 1.2$ in the COSMOS field. We compare our sample with a comprehensive compilation of data available in the literature for local and high-redshift starbursting systems and quasars. We show that the $[\mathrm{C} \mathrm{I}]\left({ }^{3} P_{1} \rightarrow{ }^{3} P_{0}\right)$ luminosity correlates on global scales with the infrared luminosity $L_{\mathrm{IR}}$, similar to low- $J \mathrm{CO}$ transitions. We report a systematic variation of $L_{\left[\mathrm{C}{ }_{1}\right]^{3} P_{1}-{ }^{3} P_{0}} / L_{\mathrm{IR}}$ as a function of the galaxy type, with the ratio being larger for main-sequence galaxies than for starbursts and submillimeter galaxies at fixed $L_{\mathrm{IR}}$. The $L_{[\mathrm{C}]^{3} P_{1}-{ }^{3} P_{0}}^{\prime} / L_{\mathrm{CO}(2-1)}^{\prime}$ and $M_{[\mathrm{C} \mathrm{I}]} / M_{\text {dust }}$ mass ratios are similar for mainsequence galaxies and for local and high-redshift starbursts within a 0.2 dex intrinsic scatter, suggesting that $[\mathrm{C} I]$ is a good tracer of molecular gas mass as $\mathrm{CO}$ and dust. We derive a fraction of $f_{[\mathrm{C} I]}=M_{\left[\mathrm{C}_{1]}\right]} / M_{\mathrm{C}} \sim 3 \%-13 \%$ of the total carbon mass in the atomic neutral phase. Moreover, we estimate the neutral atomic carbon abundance, the fundamental ingredient to calibrate $[\mathrm{C} \mathrm{I}]$ as a gas tracer, by comparing $L_{[\mathrm{CI}]^{3} P_{1}-{ }^{-3} P_{0}}$ and available gas masses from CO lines and dust emission. We find lower [C I] abundances in main-sequence galaxies than in starbursting systems and submillimeter galaxies as a consequence of the canonical $\alpha_{\mathrm{CO}}$ and gas-to-dust conversion factors. This argues against the application to different galaxy populations of a universal standard [C $\mathrm{I}]$ abundance derived from highly biased samples.
\end{abstract}

Key words: galaxies: evolution - galaxies: ISM - galaxies: high-redshift - galaxies: star formation - submillimeter: galaxies - submillimeter: ISM

Supporting material: FITS files

\section{Introduction}

The growth, structure, dynamics, and eventually fate of starforming galaxies are largely regulated by the amount of gas available to form stars and are therefore crucial parameters to estimate. While low- $J$ transitions of carbon monoxide ${ }^{12} \mathrm{CO}$ molecules and optically thin dust emission are generally trustable tracers for the molecular gas content of galaxies, they depend on the excitation conditions, metallicity, radiation field, redshift, geometry, or good photometric coverage of the farinfrared emission, and their applicability becomes progressively more observing time-consuming with increasing redshift (e.g., Magdis et al. 2012a, 2017; Bolatto et al. 2013; Carilli \& Walter 2013; Scoville et al. 2014; Papovich et al. 2016). Alternative and independent proxies are, therefore, highly valuable. These include cold molecular and photodissociation region (PDR) gas tracers, responsible for a large fraction of cooling (e.g., [C II] $\lambda 158 \mu \mathrm{m}$, Zanella et al. 2018; polycyclic aromatic hydrocarbon features (PAHs), Cortzen et al. 2019).

The submillimetric atomic carbon transitions [C I] $\left({ }^{3} P_{1} \rightarrow{ }^{3} P_{0}\right) \quad\left(\nu_{\text {rest }}=492.161 \mathrm{GHz}\right)$ and $[\mathrm{CI}] \quad\left({ }^{3} P_{2} \rightarrow{ }^{3} P_{1}\right)$ $\left(\nu_{\text {rest }}=809.344 \mathrm{GHz}\right)$ have been put forward as potential tracers of the bulk of the molecular gas in galaxies. These lines have an excitation temperature of $T_{\mathrm{ex}}=23.6$ and $62.5 \mathrm{~K}$, respectively, and a critical density for collisions with hydrogen atoms of $n_{\text {crit }} \approx 10^{3} \mathrm{~cm}^{-3}$. They can thus probe a wide range of interstellar medium (ISM) conditions, and they are normally found associated with PDRs. Early simple plane-parallel modeling of these regions, predicting [C I] just in narrow gas slabs between $\mathrm{CO}$ and $[\mathrm{C} \mathrm{II}]$, generated an initial skepticism of the real usefulness of $[\mathrm{C} \mathrm{I}]$ transitions as molecular gas tracers, recently overcome by a growing body of theoretical and observational work. Modern PDR models, including nonequilibrium chemistry 
(Stoerzer et al. 1997), turbulent mixing (Xie et al. 1995; Glover et al. 2015), clumpy geometries (Stutzki et al. 1998), and the effect of cosmic rays (Papadopoulos et al. 2004, 2018; Bisbas et al. 2015, 2017), can better explain the detection of [C I] fully concomitant with $\mathrm{CO}(1-0)$ and ${ }^{13} \mathrm{CO}$ over a wide range of conditions, with a surprisingly constant ratio $N([\mathrm{C} \mathrm{I}]) / N$ (CO) $\sim 0.1-0.2$ and tightly correlated intensities (e.g., Keene et al. 1996; Ojha et al. 2001; Ikeda et al. 2002).

The use of $[\mathrm{CI}]$ as a tracer of the molecular gas mass could even be advantageous over the traditional ${ }^{12} \mathrm{CO}$ molecule in many respects. (1) The [C I] lines are as optically thin as ${ }^{13} \mathrm{CO}$ $\left(\tau_{\left[\mathrm{C}_{\mathrm{I}}\right]} \sim 0.1-1\right.$ in giant molecular clouds in the Milky Way and its center; Ojha et al. 2001; Ikeda et al. 2002), allowing us to probe higher column densities of cold molecular gas than ${ }^{12} \mathrm{CO}$. (2) They do not suffer from the "excitation bias" affecting the high- $J\left(J_{\text {up }} \geqslant 4\right){ }^{12} \mathrm{CO}$ transitions, the latter being unable to capture cool (kinetic temperature $T_{\text {kin }}<50 \mathrm{~K}$ ) and subthermally excited gas (at densities of $n<10^{4} \mathrm{~cm}^{-3}$; Papadopoulos et al. 2004), especially in the presence of strong UV radiation fields. (3) Models show that the $[\mathrm{CI}]\left({ }^{3} P_{1}-{ }^{3} P_{0}\right)$ luminosity correlates with the total $M_{\text {gas }}$ better than both ionized carbon [C II] and $\mathrm{CO}(1-0)$, regardless of the local radiation intensity field and spanning at least four orders of magnitude in density $\left(n=10-10^{4} \mathrm{~cm}^{-3}\right.$; S. Madden et al. 2018, in preparation). (4) For the high cosmic-ray rates expected in high-redshift galaxies, $\mathrm{CO}$ is destroyed, while [C I] and [C II] become more abundant (Papadopoulos et al. 2004, 2018; Bisbas et al. 2015, 2017). (5) The simple three-level structure of $[\mathrm{CI}]$ allows for breaking the temperature-density degeneracy hampering other line tracers, and the excitation conditions of the molecular gas (i.e., its excitation temperature $T_{\text {ex }}$ ) can be straightforwardly derived from the ratio $L_{[\mathrm{C}]^{3} P_{2}-{ }^{3} P_{1}}^{\prime} / L_{[\mathrm{C} \mathrm{I}]]^{3} P_{1}-{ }^{3} P_{0}}^{\prime}$ (Weiß et al. 2003).

The [C I] $\left({ }^{3} P_{1}-{ }^{3} P_{0}\right)$ and [C I] $\left({ }^{3} P_{2}-{ }^{3} P_{1}\right)$ transitions have been detected in molecular clouds of the Galactic disk, the Galactic center, and other nearby galaxies (Stutzki et al. 1997; Gerin \& Phillips 2000; Ojha et al. 2001; Ikeda et al. 2002) for some early measurements (see Israel et al. 2015; Kamenetzky et al. 2016; Jiao et al. 2017; Lu et al. 2017, among others, for more recent compilations). At high redshift, the detections reported so far include strongly starbursting submillimeter galaxies (SMGs), radio galaxies, and quasar hosts (QSOs), often gravitationally lensed and magnified up to factor of $30 \times$ (e.g., Walter et al. 2011; Alaghband-Zadeh et al. 2013; Gullberg et al. 2016; Bothwell et al. 2017; Popping et al. 2017; Yang et al. 2017; Andreani et al. 2018; Banerji et al. 2018; Emonts et al. 2018; Oteo et al. 2018 and references therein). First results seem to indicate that in these galaxies, the excitation properties of the interstellar medium and the [C I] abundances are similar or more extreme than in (ultra)luminous infrared galaxies and starbursts in the local universe. Nevertheless, despite being a valuable reference sample, this handful of galaxies ( 35) selected in diverse ways is not representative of the average main-sequence population (e.g., Daddi et al. 2007; Elbaz et al. 2007; Noeske et al. 2007; Magdis et al. 2010), thus strongly biasing the general conclusions we can draw about the bulk of high-redshift galaxies. In order to remedy this situation, we designed and carried out a survey with the Atacama Large Millimeter Array (ALMA) targeting [CI] $\left({ }^{3} P_{1}-{ }^{3} P_{0}\right)$ in upper main-sequence galaxies in the COSMOS field at $z \sim 1.2$ (see Bourne et al. 2018 for a recent similar effort). Here we present the first results of a sample of objects with secure [C I] $\left({ }^{3} P_{1}-{ }^{3} P_{0}\right)$, dust continuum, and/or
$\mathrm{CO}(2-1)$ determinations, starting with exploring the potential use of [C I] $\left({ }^{3} P_{1}-{ }^{3} P_{0}\right)$ as an effective tracer of the molecular gas on global scales in normal main-sequence galaxies.

This work is structured as follows. In Section 2, we describe how we selected the sample of main-sequence galaxies and the available data; in particular, in Section 2.3, we present the new ALMA data targeting [C I] $\left({ }^{3} P_{1}-{ }^{3} P_{0}\right)$, along with results from independent programs targeting other $\mathrm{CO}$ lines for the same objects. In Section 3, we compile data from the literature to build a comparison sample for our sources. Section 4 contains the analysis and main results of this work, followed by a discussion in Section 5 and the conclusions in Section 6. Unless stated otherwise, we assume a $\Lambda$ CDM cosmology with $\Omega_{\mathrm{m}}=0.3, \Omega_{\Lambda}=0.7$, and $H_{0}=70 \mathrm{~km} \mathrm{~s}^{-1} \mathrm{Mpc}^{-1}$ and a Chabrier initial mass function (IMF; Chabrier 2003). All magnitudes are expressed in the $\mathrm{AB}$ system. All of the literature data have been homogenized with our conventions.

\section{Sample and Observations}

\subsection{Sample Selection}

We selected targets in the COSMOS field (Scoville et al. 2007) with (1) an available stellar mass estimate (Muzzin et al. 2013; Laigle et al. 2016), (2) a spectroscopic confirmation with optical or near-infrared spectrographs from the COSMOS master catalog (M. Salvato et al. 2018, in preparation), and (3) a Herschel/PACS 100 and/or $160 \mu \mathrm{m}>3 \sigma$ detection in the publicly available PEP catalog (Lutz et al. 2011). The latter requirement resulted in the selection of massive galaxies mainly on the upper main sequence and with mean dust temperatures of $T_{\text {dust }} \gtrsim 30 \mathrm{~K}$. We further chose sources at $z_{\mathrm{opt} / \mathrm{NIR}}=1.05-1.63$ to maximize the overlap with parallel and independent ALMA programs targeting $\mathrm{CO}(2-1)$ and CO (5-4) (Section 2.3; E. Daddi et al. 2018, in preparation). These criteria drove to an initial pool of 204 sources. We then grouped the sources to maximize the number of targets observable in three frequency configurations of ALMA Band 6 . This resulted in the final selection of 50 sources in the redshift ranges $z_{\mathrm{opt} / \mathrm{NIR}}=1.09-1.18$ and 1.23-1.32, randomly sampling the whole interval of total infrared luminosities of the original parent sample. The first redshift interval allowed us to simultaneously cover [C I] $\left({ }^{3} P_{1}-{ }^{3} P_{0}\right)$ and the $\mathrm{CO}(4-3)$ transition $\left(\nu_{\text {rest }}=461.041 \mathrm{GHz}\right.$; Section 2.3$)$.

Here we present the results for 21 sources with both (1) an unambiguous spectroscopic confirmation from a submillimeter transition and (2) an estimate of the $\mathrm{CO}(2-1)$ flux and/or of the dust continuum emission, so to ensure at least one molecular gas mass determination with a standard method (Section 4.4). The former criterion allows us to confidently measure even weak $[\mathrm{C} \mathrm{I}]\left({ }^{3} P_{1}-{ }^{3} P_{0}\right)$ fluxes or put stringent upper limits at the expected line location. In fact, a nondetection could be due to either intrinsic weak [C I] $\left({ }^{3} P_{1}-{ }^{3} P_{0}\right)$ emission or the absence of frequency coverage owing to inaccurate redshifts. Significant offsets between optical/near-infrared and submillimeter redshift estimates are not unusual, especially considering the heterogeneous original data catalogs in the literature and the different approaches to assessing the redshift quality. Some of the sources we selected did have initial low-quality flags in the COSMOS compilation. The requirement of alternative gas tracers excludes $2 / 29$ extra sources with a single [C I] $\left({ }^{3} P_{1}-{ }^{3} P_{0}\right)$ line detection and no dust continuum or any $\mathrm{CO}$ emission covered. The rest of the sample not analyzed here is 


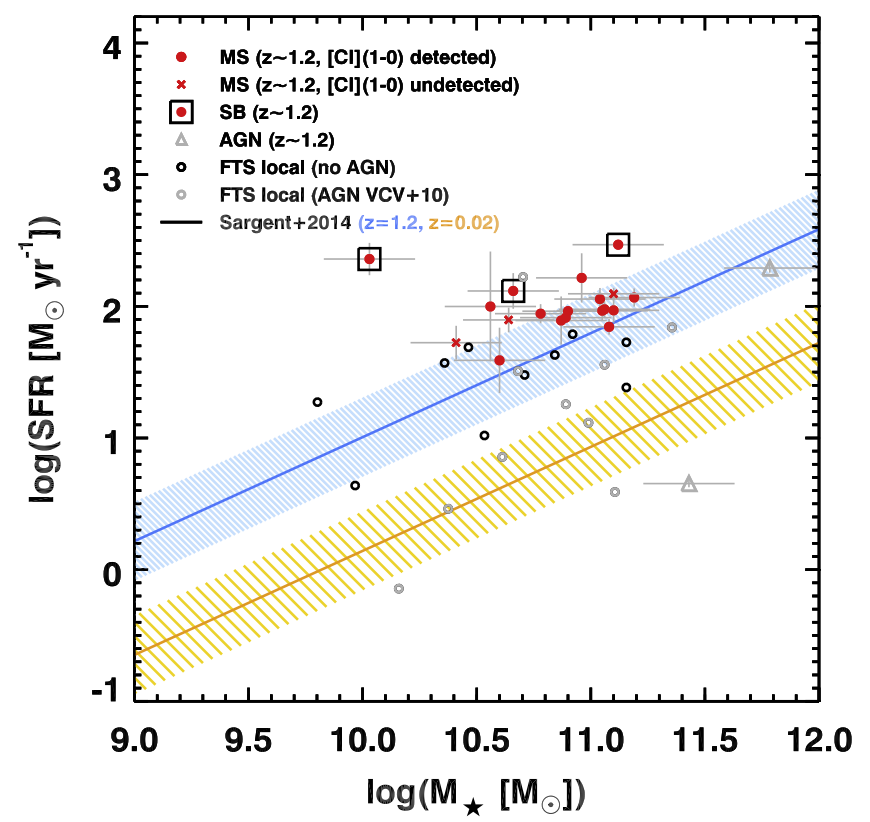

Figure 1. Stellar mass-SFR plane. Red filled circles and crosses mark our sample of MS galaxies at $z \sim 1.2$ with and without a $>3 \sigma[\mathrm{C} \mathrm{I}]\left({ }^{3} P_{1}-{ }^{3} P_{0}\right)$ detection, respectively. Black squares indicate starbursts. Gray triangles mark the positions of the two AGN-dominated galaxies in our sample. Open black and gray circles represent the local comparison sample of [C I] $\left({ }^{3} P_{1}-{ }^{3} P_{0}\right)$-detected LIRGs and active galaxies, respectively (Liu et al. 2015). The blue (orange) solid line and hatched area mark the position of the main sequence at $z=1.2(z=0.02)$ as parameterized by Sargent et al. (2014) and its $1 \sigma$ scatter of 0.3 dex.

characterized by several things, as follows. (1) Good coverage of the far-infrared spectral energy distribution (SED) but lowquality optical/near-infrared spectra and no submillimeter line detection (7/29 sources) or ascertained wrong redshifts from $\mathrm{CO}$ lines that became available after our ALMA observations $(7 / 29$ objects). In the latter case, the [C I] $\left({ }^{3} P_{1}-{ }^{3} P_{0}\right)$ transition fell outside the covered frequency range or could not be identified unambiguously. (2) No detections in three or more followed-up submillimeter lines (4/29 galaxies), despite good quality flags associated with $z_{\mathrm{opt} / \mathrm{NIR}}$ and good coverage of the far-infrared SED. This might be due to a wrong association with an optical/ near-infrared counterpart or redshift quality assessment. (3) Good quality $z_{\mathrm{opt} / \mathrm{NIR}}$ but serious blending and source misidentification in the far-infrared and submillimeter bands, which we could verify only a posteriori with new catalogs becoming available (3/29 sources). (4) A combination of low-quality $z_{\text {opt/NIR }}$ and bad coverage or even nondetection in the far-infrared SED based on the new catalogs superseding the previous compilations $(6 / 29$ galaxies). For these galaxies, the absence of or a spectroscopic confirmation from a submillimeter line and/or an alternative gas tracer securely detected, either dust or $\mathrm{CO}(2-1)$, does not allow a proper assessment of [C I] $\left({ }^{3} P_{1}-{ }^{3} P_{0}\right)$ as a proxy for the molecular gas content in our sample.

Respectively, $100 \%$ and $95 \%$ of the 21 sources presented here are detected with a cumulative infrared signal-to-noise ratio $(\mathrm{S} / \mathrm{N})>3$ and $>5$ in the "super-deblended" catalog by Jin et al. (2018; see below). The latter became available after our ALMA observations and superseded the PEP catalog in our analysis. Moreover, 16/21 galaxies lie on the main sequence at their redshift, $3 / 21$ are classified as starbursts $(>3.5 \times$ above the main sequence), and $2 / 21$ suffer from a significant contribution from active galactic nucleus (AGN) emission
(Figure 1). The main-sequence galaxies are, on average, $\sim 1.8 \times$ above the parameterization by Sargent et al. (2014), as expected from our selection.

A discussion of the whole sample and the detailed combined modeling of all the $\mathrm{CO}$ and [C I] transitions is postponed to a dedicated forthcoming paper (F. Valentino et al. 2018, in preparation).

\subsection{Ancillary Data}

Our sample benefits from the excellent photometric and spectroscopic coverage available in COSMOS. In particular, we adopted the stellar masses listed in Laigle et al. (2016), consistent with the values derived by Muzzin et al. (2013), both modeling the UV-to-near-infrared SEDs with standard recipes. Moreover, we modeled the "super-deblended" infrared photometry Jin et al. (2018) as in Magdis et al. (2012b) to derive the galaxy-integrated far-infrared properties. The "super-deblending" of the highly confused far-infrared bands is based on an active choice of the radio and $24 \mu \mathrm{m}$ priors based on the galaxies' spectral properties, reducing the blending degeneracies and resulting in well-behaved flux density uncertainties (see Liu et al. 2018 for a detailed description of the method). Whenever available, we fit the emission from Spitzer/MIPS $24 \mu \mathrm{m}$ (Sanders et al. 2007), the Herschel/PACS (Lutz et al. 2011) and SPIRE bands (Oliver et al. 2012), JCMT/SCUBA2 (Geach et al. 2017), ASTE/AzTEC (Aretxaga et al. 2011), and IRAM/MAMBO (Bertoldi et al. 2007), as well as the ALMA continuum emission at $\sim 1.1-1.3 \mathrm{~mm}$ (Section 2.3), with an expansion of the Draine \& Li (2007, hereafter DL07) model library (Figure 7 in the Appendix). We further included a dusty torus component surrounding the AGN following Mullaney et al. (2011) and subtracted this contribution from the total $L_{\mathrm{IR}}$ we derived. Therefore, $L_{\mathrm{IR}}$ always refers only to the component due to star formation for this sample. We then converted $L_{\mathrm{IR}}$ into star formation rate (SFR) as $\mathrm{SFR}=$ $L_{\mathrm{IR}}\left[L_{\odot}\right] /\left(9.86 \times 10^{9}\right) M_{\odot} \mathrm{yr}^{-1}$ (Kennicutt $1998 \mathrm{a}$; converted to a Chabrier IMF). The emission from the dusty torus is relevant ( $\sim 40 \%$ and $>95 \%$ of $L_{\mathrm{IR}}$ ) in two sources, flagged everywhere as "AGN" hereafter. Moreover, their stellar masses are likely overestimated, due to significant AGN emission in the nearinfrared bands. Therefore, for the purpose of this work, we will not include the AGN in any further step of the analysis. While fitting the SEDs, we included the upper limits in every band, modeling the nominal values weighted by their large uncertainties. We then bootstrapped the values within the observed errors to estimate the statistical uncertainties on the derived quantities. In Tables 1 and 2, we report the 8-1000 $\mu \mathrm{m}$ total $L_{\mathrm{IR}}$ for all of our galaxies, the contribution from dusty tori, and the total dust mass $M_{\text {dust }}$. We note that $L_{\mathrm{IR}}$ is well constrained for the vast majority of our sample, while $M_{\text {dust }}$ critically relies on the availability of a measurement in the Rayleigh-Jeans tail of the dust emission.

\subsection{ALMA [CI] (1-0) and CO Observations}

We collected ALMA Band 6 observations during Cycle 4 (Project ID: 2016.1.01040.S, PI: F. Valentino). Galaxies were grouped in three scheduling blocks targeting [C I] $\left({ }^{3} P_{1}-{ }^{3} P_{0}\right)$ $\left(\nu_{\text {rest }}=492.161 \mathrm{GHz}\right)$ and $\mathrm{CO}(4-3)\left(\nu_{\text {rest }}=461.041 \mathrm{GHz}\right)$ at $z \sim 1.15$ and $[\mathrm{C} \mathrm{I}]\left({ }^{3} P_{1}-{ }^{3} P_{0}\right)$ only at $z \sim 1.28$ within contiguous spectral windows of $1.875 \mathrm{GHz}$ and a requested spectral resolution of $7.8 \mathrm{MHz}\left(\sim 10 \mathrm{~km} \mathrm{~s}^{-1}\right)$. Two out of three of the blocks were fully observed, while the third was 
Table 1

Main-sequence Galaxies at $z \sim 1.2$

\begin{tabular}{|c|c|c|c|c|c|c|c|c|c|c|c|c|}
\hline (1) & $\begin{array}{l}\text { R.A. } \\
\text { (deg) } \\
\text { (2) }\end{array}$ & $\begin{array}{l}\text { Decl. } \\
\text { (deg) } \\
\text { (3) }\end{array}$ & (4) & $\begin{array}{c}\log \left(M_{\star}\right) \\
\left(M_{\odot}\right) \\
(5)\end{array}$ & $\begin{array}{c}L_{\mathrm{IR}} \\
\left(10^{11} L_{\odot}\right) \\
(6)\end{array}$ & $\begin{array}{c}L_{\mathrm{FIR}} \\
\left(10^{11} L_{\odot}\right) \\
(7)\end{array}$ & $\begin{array}{c}L_{[\mathrm{[C} \mathrm{I}]^{3} P_{1}-{ }^{3} P_{0}}^{\prime} \\
\left(10^{10} \mathrm{Km} \mathrm{s}^{-1} \mathrm{pc}^{2}\right) \\
(8)\end{array}$ & $\begin{array}{c}L_{\mathrm{CO}(2-1)}^{\prime} \\
\left(10^{10} \mathrm{~K} \mathrm{~km} \mathrm{~s}^{-1} \mathrm{pc}^{2}\right) \\
(9)\end{array}$ & $\begin{array}{c}I_{[\mathrm{C} \mathrm{I}]^{3} P_{1}-{ }^{3} P_{0}} \\
\left(\mathrm{Jy} \mathrm{km} \mathrm{s}^{-1}\right) \\
(10)\end{array}$ & $\begin{array}{c}I_{\mathrm{CO}(2-1)} \\
\left(\mathrm{Jy} \mathrm{km} \mathrm{s}^{-1}\right) \\
(11)\end{array}$ & $\begin{array}{r}f_{\mathrm{AGN}} \\
(12)\end{array}$ & (13) \\
\hline 4233 & 150.39732 & 2.24088 & $1.1630 \pm 0.0003^{\mathrm{a}}$ & 10.89 & $8.09 \pm 0.49$ & $5.05 \pm 0.30$ & $0.24 \pm 0.05$ & $\cdots$ & $0.6 \pm 0.14$ & $\cdots$ & $\cdots$ & MS \\
\hline 7540 & 150.41281 & 1.85157 & $1.1714 \pm 0.0003^{\mathrm{a}}$ & 11.06 & $9.36 \pm 1.32$ & $7.36 \pm 1.04$ & $0.34 \pm 0.08$ & & $0.86 \pm 0.19$ & $\ldots$ & 0.12 & MS \\
\hline 13205 & 150.57484 & 1.94786 & $1.2660 \pm 0.0004^{\mathrm{b}}$ & 11.10 & $12.28 \pm 1.27$ & $9.68 \pm 1.00$ & $<0.2$ & $1.93 \pm 0.39$ & $<0.44$ & $0.92 \pm 0.18$ & 0.16 & MS \\
\hline 13250 & 150.17650 & 1.95523 & $1.1484 \pm 0.0002^{\mathrm{a}}$ & 10.41 & $5.22 \pm 1.54$ & $3.68 \pm 1.09$ & $<0.1$ & $\ldots$ & $<0.26$ & $\ldots$ & 0.04 & MS \\
\hline 18538 & 150.12639 & 2.43285 & $1.2694 \pm 0.0001^{\mathrm{c}}$ & 10.56 & $9.83 \pm 9.45$ & $8.22 \pm 7.91$ & $0.19 \pm 0.02$ & $\ldots$ & $0.41 \pm 0.05$ & $\ldots$ & 0.01 & MS \\
\hline 18911 & 150.04820 & 2.46767 & $1.1709 \pm 0.0003^{\mathrm{a}}$ & 10.64 & $7.78 \pm 1.68$ & $5.87 \pm 1.27$ & $<0.16$ & $0.58 \pm 0.13$ & $<0.39$ & $0.32 \pm 0.07$ & 0.04 & MS \\
\hline 19021 & 150.05878 & 2.47739 & $1.2581 \pm 0.0003^{\mathrm{d}}$ & 11.78 & $19.27 \pm 1.57$ & $14.57 \pm 1.19$ & $0.35 \pm 0.07$ & $1.90 \pm 0.24$ & $0.76 \pm 0.14$ & $0.91 \pm 0.11$ & 0.41 & AGN \\
\hline 26925 & 150.46350 & 1.88331 & $1.1671 \pm 0.0003^{\mathrm{a}}$ & 11.05 & $9.13 \pm 1.66$ & $7.56 \pm 1.37$ & $0.35 \pm 0.06$ & $1.47 \pm 0.22$ & $0.88 \pm 0.16$ & $0.81 \pm 0.12$ & 0.08 & MS \\
\hline 30694 & 149.66026 & 2.41099 & $1.1606 \pm 0.0002^{\mathrm{a}}$ & 10.78 & $8.67 \pm 1.45$ & $7.58 \pm 1.26$ & $0.24 \pm 0.03$ & $1.62 \pm 0.21$ & $0.61 \pm 0.09$ & $0.91 \pm 0.12$ & 0.32 & MS \\
\hline 32394 & 150.06781 & 1.85108 & $1.1345 \pm 0.0001^{\mathrm{a}}$ & 10.03 & $22.54 \pm 6.37$ & $12.90 \pm 3.65$ & $0.24 \pm 0.05$ & $\ldots$ & $0.64 \pm 0.12$ & $\ldots$ & 0.06 & SB \\
\hline 35349 & 150.49902 & 1.72446 & $1.2543 \pm 0.0002^{\mathrm{c}}$ & 11.19 & $11.5 \pm 1.83$ & $8.65 \pm 1.38$ & $0.41 \pm 0.11$ & $3.57 \pm 0.44$ & $0.89 \pm 0.25$ & $1.72 \pm 0.21$ & 0.07 & MS \\
\hline 36053 & 149.68564 & 1.91986 & $1.1573 \pm 0.0003^{\mathrm{a}}$ & 10.87 & $7.69 \pm 3.24$ & $5.38 \pm 2.26$ & $0.17 \pm 0.05$ & $\ldots$ & $0.43 \pm 0.13$ & $\ldots$ & 0.04 & MS \\
\hline 36945 & 150.19550 & 2.12404 & $1.1569 \pm 0.0003^{\mathrm{a}}$ & 11.43 & $0.44 \pm 0.03$ & $0.37 \pm 0.02$ & $0.17 \pm 0.05$ & $1.31 \pm 0.37$ & $0.44 \pm 0.14$ & $0.47 \pm 0.13$ & 0.99 & AGN \\
\hline 37250 & 149.61813 & 2.19346 & $1.1526 \pm 0.0002^{\mathrm{c}}$ & 10.96 & $16.21 \pm 6.97$ & $12.48 \pm 5.37$ & $0.68 \pm 0.06$ & $4.58 \pm 0.32$ & $1.76 \pm 0.16$ & $2.6 \pm 0.18$ & 0.03 & MS \\
\hline 37508 & 150.38953 & 2.25585 & $1.3020 \pm 0.0003^{\mathrm{b}}$ & 11.04 & $11.20 \pm 2.17$ & $6.38 \pm 1.24$ & $0.31 \pm 0.08$ & $1.48 \pm 0.42$ & $0.64 \pm 0.16$ & $0.43 \pm 0.12$ & 0.12 & MS \\
\hline 38053 & 150.55685 & 2.39140 & $1.1562 \pm 0.0003^{\mathrm{a}}$ & 10.66 & $12.90 \pm 4.03$ & $10.84 \pm 3.38$ & $0.26 \pm 0.05$ & $1.69 \pm 0.31$ & $0.67 \pm 0.14$ & $0.96 \pm 0.17$ & 0.03 & SB \\
\hline 44641 & 150.65852 & 2.31786 & $1.1495 \pm 0.0002^{\mathrm{a}}$ & 11.10 & $9.19 \pm 1.99$ & $7.72 \pm 1.67$ & $0.44 \pm 0.06$ & $1.63 \pm 0.35$ & $1.16 \pm 0.17$ & $0.93 \pm 0.20$ & 0.07 & MS \\
\hline $121546^{\mathrm{e}}$ & 150.05910 & 2.21995 & $1.1392 \pm 0.0002^{\mathrm{a}}$ & 11.08 & $6.89 \pm 0.94$ & $5.28 \pm 0.72$ & $0.39 \pm 0.04$ & $\ldots$ & $1.04 \pm 0.12$ & $\ldots$ & 0.16 & MS \\
\hline 188090 & 150.42799 & 2.55860 & $1.2383 \pm 0.0002^{\mathrm{c}}$ & 11.12 & $28.97 \pm 1.22$ & $22.74 \pm 0.96$ & $0.23 \pm 0.04$ & $\ldots$ & $0.51 \pm 0.09$ & $\ldots$ & 0.22 & SB \\
\hline 192337 & 150.42073 & 2.62297 & $1.2884 \pm 0.0003^{\mathrm{c}}$ & 10.90 & $9.06 \pm 0.64$ & $7.50 \pm 0.53$ & $0.30 \pm 0.07$ & $\cdots$ & $0.62 \pm 0.14$ & $\cdots$ & - & MS \\
\hline 218445 & 149.92007 & 2.61905 & $1.1199 \pm 0.0004^{\mathrm{c}}$ & 10.60 & $3.83 \pm 2.19$ & $3.25 \pm 1.86$ & $0.49 \pm 0.09$ & $\cdots$ & $1.33 \pm 0.25$ & $\cdots$ & 0.04 & MS \\
\hline
\end{tabular}

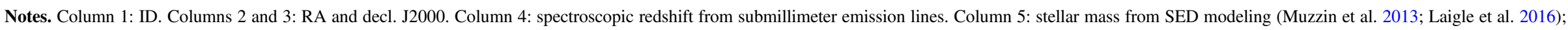

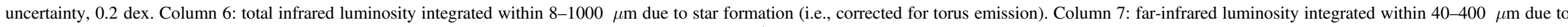

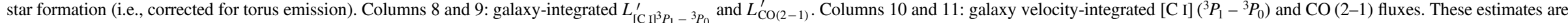

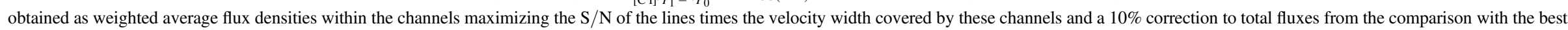

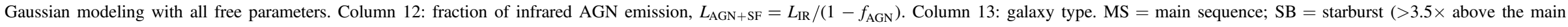
sequence); $\mathrm{AGN}=$ SED dominated by torus emission.

${ }^{\mathrm{a}}$ From $\mathrm{CO}(4-3)$.

${ }^{\mathrm{b}} \mathrm{CO}(5-4)$.

${ }^{\mathrm{c}}\left[\mathrm{C}\right.$ I] $\left({ }^{3} P_{1}-{ }^{3} P_{0}\right)$.

${ }^{\mathrm{d}} \mathrm{CO}(2-1)$;

${ }^{\mathrm{e}}$ Selected based on $z_{\text {opt/NIR }}$ from Casey et al. (2012). Upper limits are at $3 \sigma$.

(This table is available in its entirety in FITS format.) 
Table 2

Main-sequence Galaxies at $z \sim 1.2$

\begin{tabular}{|c|c|c|c|c|c|c|}
\hline (1) & $\begin{array}{c}M_{\text {dust }} \\
\left(10^{8} M_{\odot}\right) \\
(2)\end{array}$ & $\begin{array}{c}\delta_{\mathrm{GDR}} \\
(3)\end{array}$ & $\begin{array}{l}\alpha_{\mathrm{CO}} \\
(4)\end{array}$ & $\begin{array}{c}M_{[\mathrm{C} \mathrm{I}]} \\
\left(10^{6} M_{\odot}\right) \\
(5)\end{array}$ & $\begin{array}{c}{[\mathrm{C} \mathrm{I}] /\left[\mathrm{H}_{2}\right]_{(\mathrm{dust})}} \\
\left(\times 10^{-5}\right) \\
(6)\end{array}$ & $\begin{array}{c}{[\mathrm{C} \mathrm{I}] /\left[\mathrm{H}_{2}\right]_{(\mathrm{CO})}} \\
\left(\times 10^{-5}\right) \\
(7)\end{array}$ \\
\hline 4233 & $5.8 \pm 1.1$ & 82.0 & $\cdots$ & $3.0 \pm 0.7$ & $1.4 \pm 0.8$ & $\ldots$ \\
\hline 7540 & $7.7 \pm 1.2$ & 72.5 & $\cdots$ & $4.3 \pm 0.9$ & $1.7 \pm 0.9$ & $\ldots$ \\
\hline 13205 & $2.3 \pm 0.3^{\mathrm{a}}$ & 72.3 & 2.6 & $<2.5$ & $<3.4$ & $<1.0$ \\
\hline 13250 & $2.6 \pm 0.7$ & 121.5 & $\ldots$ & $<1.3$ & $<0.9$ & $\ldots$ \\
\hline 18538 & $3.5 \pm 0.5$ & 114.3 & $\ldots$ & $2.4 \pm 0.3$ & $1.4 \pm 0.7$ & $\ldots$ \\
\hline 18911 & $1.2 \pm 0.2^{\mathrm{a}}$ & 102.5 & 4.2 & $<1.9$ & $<3.7$ & $<1.5$ \\
\hline 19021 & $5.7 \pm 0.5$ & 76.3 & 2.8 & $4.3 \pm 0.8$ & $2.3 \pm 1.1$ & $1.6 \pm 0.8$ \\
\hline 26925 & $4.6 \pm 0.7$ & 72.8 & 2.6 & $4.4 \pm 0.8$ & $2.9 \pm 1.5$ & $2.2 \pm 1.1$ \\
\hline 30694 & $4.2 \pm 0.4$ & 91.2 & 3.6 & $3.0 \pm 0.4$ & $1.8 \pm 0.9$ & $1.0 \pm 0.5$ \\
\hline 32394 & $7.6 \pm 1.6$ & 30.0 & $\ldots$ & $3.0 \pm 0.6$ & $3.0 \pm 1.6$ & $\ldots$ \\
\hline 35349 & $12.0 \pm 2.8$ & 76.3 & 2.8 & $5.1 \pm 1.4$ & $1.3 \pm 0.7$ & $1.0 \pm 0.5$ \\
\hline 36053 & $3.2 \pm 0.7$ & 82.9 & $\ldots$ & $2.1 \pm 0.6$ & $1.8 \pm 1.1$ & $\ldots$ \\
\hline 36945 & $4.0 \pm 0.7$ & 76.3 & 2.8 & $2.2 \pm 0.7$ & $1.6 \pm 0.9$ & $1.1 \pm 0.7$ \\
\hline 37250 & $9.6 \pm 0.8$ & 83.9 & 3.2 & $8.5 \pm 0.8$ & $2.4 \pm 1.1$ & $1.1 \pm 0.5$ \\
\hline 37508 & $0.9 \pm 0.1^{\mathrm{a}}$ & 75.1 & 2.7 & $3.9 \pm 1.0$ & $12.6 \pm 6.6$ & $1.8 \pm 1.1$ \\
\hline 38053 & $3.1 \pm 0.4$ & 30.0 & 0.8 & $3.3 \pm 0.7$ & $8.0 \pm 4.2$ & $4.6 \pm 2.5$ \\
\hline 44641 & $7.0 \pm 0.7$ & 70.2 & 2.5 & $5.5 \pm 0.8$ & $2.6 \pm 1.3$ & $2.6 \pm 1.4$ \\
\hline 121546 & $2.7 \pm 0.7^{b}$ & 69.1 & $\ldots$ & $4.9 \pm 0.6$ & $6.0 \pm 3.2$ & $\ldots$ \\
\hline 188090 & $8.4 \pm 0.8$ & 30.0 & $\ldots$ & $2.8 \pm 0.5$ & $2.6 \pm 1.3$ & $\ldots$ \\
\hline 192337 & $4.6 \pm 0.6$ & 82.4 & $\ldots$ & $3.7 \pm 0.8$ & $2.2 \pm 1.2$ & $\ldots$ \\
\hline 218445 & $6.4 \pm 1.7$ & 97.0 & $\ldots$ & $6.1 \pm 1.1$ & $2.2 \pm 1.2$ & $\ldots$ \\
\hline
\end{tabular}

Notes. Column 1: ID. Column 2: dust mass from SED modeling. Column 3: gas-to-dust conversion factor. Value fixed to $\delta_{\mathrm{GDR}}=30$ for starbursts. Uncertainty 0.2 dex. Column 4: $\alpha_{\mathrm{CO}}$ conversion factor. Value fixed to $\alpha_{\mathrm{CO}}=0.8 M_{\odot} /\left(\mathrm{K} \mathrm{km} \mathrm{s}^{-1} \mathrm{pc}^{2}\right)$ for starbursts. Uncertainty 0.2 dex. Column 5: mass of [C I] (from $L_{[\mathrm{C}]^{3} P_{1}-{ }^{3} P_{0}}^{\prime}$ assuming $\left.T_{\mathrm{ex}}=30 \mathrm{~K}\right)$. Column 6: atomic carbon abundance adopting $M_{\text {gas }}($ dust $)$ and removing the $1.36 \times$ contribution of helium. Column 7: atomic carbon abundance adopting $M_{\text {gas }}(\mathrm{CO})$ and removing the $1.36 \times$ contribution of helium. Upper limits are $<3 \sigma$. Systematic uncertainties on $M_{\text {gas }}$ are not included in the error budget.

a No continuum detection in the Rayleigh-Jeans tail of the dust emission. This value should be treated as an order-of-magnitude estimate.

${ }^{\mathrm{b}}$ Significant blending of the photometry.

(This table is available in its entirety in FITS format.)

incomplete, resulting in a higher rms. Data were collected in configuration C40-1, corresponding to a synthesized beam of $2.0 \times 1$." 7 . Galaxies are generally not (or marginally) resolved, ensuring minimal flux losses. We reduced the raw data with the standard ALMA pipeline with CASA (McMullin et al. 2007). We then converted the calibrated data cubes to uvfits and analyzed them with GILDAS (Guilloteau \& Lucas 2000). We extracted 1D spectra using PSFs and circular Gaussian models and fitting visibilities in the $u v$ space with the iterative process described in Daddi et al. (2015). The [C I] $\left({ }^{3} P_{1}-{ }^{3} P_{0}\right)$ spectra are shown in Figure 7 in the Appendix. We looked for emission lines scanning the $\mathrm{S} / \mathrm{N}$ spectrum. We measured fluxes as the weighted average flux density within the channels maximizing the $\mathrm{S} / \mathrm{N}$ times the velocity width covered by these channels. We further fit single or double Gaussians to the line profile to estimate total fluxes, generally $\sim 10 \%$ larger than the fluxes measured over the number of channels maximizing the $S / N$. We finally adopted the first approach, applying a correction of $10 \%$ to the line fluxes. When multiple lines were available, we measured fluxes and upper limits on the same velocity width of the brightest line. These results agree with measurements leaving each line center and width free to vary. We measured integrated [CI] $\left({ }^{3} P_{1}-{ }^{3} P_{0}\right)$ fluxes at $>3 \sigma$ in $18 / 21$ sources down to an average rms per beam of $\sim 0.15 \mathrm{Jy} \mathrm{km} \mathrm{s}^{-1}$ for a line width of $400 \mathrm{~km} \mathrm{~s}^{-1}$ and a final velocity resolution of $\sim 20-40 \mathrm{~km} \mathrm{~s}^{-1}$. Fifteen out of 21 sources have [C I] detections significant at $>4 \sigma$. All of the remaining sources have either one or multiple $\mathrm{CO}$ lines detected at $>4 \sigma$ at the same redshift (see next paragraph), allowing us to explore the $\mathrm{S} / \mathrm{N}<4$ regime or to put secure upper limits on [C I]. We simultaneously measured the continuum emission at observed $\sim 1.3 \mathrm{~mm}$ over $7.5 \mathrm{GHz}$ assuming an intrinsic slope of $\nu^{3.5}(\beta=1.5)$. We detected significant continuum emission at $3 \sigma$ in $14 / 21$ sources down to an rms of $\sim 0.07 \mathrm{mJy}$ on the full frequency range.

From the same observing campaign, we similarly measured $\mathrm{CO}(4-3)$ fluxes at $>4 \sigma$ significance in all 14 galaxies with frequency coverage of this line. Moreover, 15 and 11 galaxies of our sample have been observed by ALMA Band 6 and 3 independent observations targeting $\mathrm{CO}(5-4)$ and $\mathrm{CO}(2-1)$, respectively (Project IDs: 2015.1.00260.S, 2016.1.00171.S, PI: Daddi; E. Daddi et al. 2018, in preparation). Data were collected at similar spatial resolutions and reduced and analyzed as we described above. For the purpose of the present work, we used high-S/N CO emissions to (1) fix the center of the circular Gaussian or PSF to extract the spectrum, central line frequency, and width of $[\mathrm{C} \mathrm{I}]\left({ }^{3} P_{1}-{ }^{3} P_{0}\right) ;(2)$ measure gas masses from $\mathrm{CO}(2-1)$; and (3) measure millimeter continuum emission. We do not find evidence of systematically broader or narrower [C I] lines than $\mathrm{CO}$ transitions, when the velocity width is left free to vary. We significantly detected $\mathrm{CO}(2-1)$ and $\mathrm{CO}(5-4)$ in all 11 and 15 targeted galaxies and continuum emission at the observed $\sim 3-1.1 \mathrm{~mm}$ for $1 / 11$ and $8 / 15$ galaxies covered by Band 3 and 6 observations, respectively. This brings the overall number of sources with $\sim 1^{\prime \prime}$ spatial resolution millimeter continuum detection to $17 / 21$. 
Table 3

Local Galaxies

\begin{tabular}{|c|c|c|c|c|c|c|c|c|c|c|c|c|}
\hline (1) & $\begin{array}{c}D_{\mathrm{L}} \\
(\mathrm{Mpc}) \\
(2)\end{array}$ & $\begin{array}{l}z_{\text {spec }} \\
(3)\end{array}$ & $\begin{array}{c}\log \left(M_{\star}\right) \\
\left(M_{\odot}\right) \\
(4)\end{array}$ & $\begin{array}{l}L_{\mathrm{IR}} \\
\left(10^{11} L_{\odot}\right) \\
(5)\end{array}$ & $\begin{array}{c}L_{[\mathrm{C} \mathrm{I}]^{3} P_{1}-{ }^{3} P_{0}}^{\prime} \\
\left(10^{8} \mathrm{Km} \mathrm{s}^{-1} \mathrm{pc}^{2}\right) \\
(6)\end{array}$ & $\begin{array}{c}L_{\mathrm{CO}(1-0)}^{\prime} \\
\left(10^{8} \mathrm{Km} \mathrm{s}^{-1} \mathrm{pc}^{2}\right) \\
(7)\end{array}$ & $\begin{array}{c}L_{\mathrm{CO}(2-1)}^{\prime} \\
\left(10^{8} \mathrm{~K} \mathrm{~km} \mathrm{~s}^{-1} \mathrm{pc}^{2}\right) \\
(8)\end{array}$ & $\begin{array}{c}I_{[\mathrm{C} \mathrm{I}]^{3} P_{1}-{ }^{3} P_{0}} \\
\left(10^{8} \mathrm{Km} \mathrm{s}^{-1} \mathrm{pc}^{2}\right) \\
(9)\end{array}$ & $\begin{array}{c}I_{\mathrm{CO}(1-0)} \\
\left(10^{2} \mathrm{Jy} \mathrm{km} \mathrm{s}^{-1}\right) \\
(10)\end{array}$ & $\begin{array}{c}I_{\mathrm{CO}(2-1)} \\
\left(10^{2} \mathrm{Jy} \mathrm{km} \mathrm{s}^{-1}\right) \\
(11)\end{array}$ & $\begin{array}{l}\eta_{\text {beam }} \\
(12)\end{array}$ & AGN \\
\hline Arp193 & 101.6 & 0.02330 & 10.5 & $4.8 \pm 1.0$ & $10.4 \pm 3.3$ & $39.4 \pm 4.2$ & $35.3 \pm 5.5$ & $7.7 \pm 2.4$ & $1.6 \pm 0.2$ & $5.7 \pm 0.9$ & 1 & $\mathrm{~N}$ \\
\hline Arp220 & 78.7 & 0.01813 & 10.7 & $16.4 \pm 3.3$ & $13.8 \pm 2.8$ & $81.0 \pm 4.5$ & $77.9 \pm 15.7$ & $16.9 \pm 3.4$ & $5.4 \pm 0.3$ & $20.9 \pm 4.2$ & 1.21 & $\mathrm{Y}$ \\
\hline Arp299-A & 44.9 & 0.01041 & $\cdots$ & $2.9 \pm 0.6$ & $4.8 \pm 1.2$ & $28.4 \pm 3.2$ & $30.1 \pm 2.4$ & $17.8 \pm 4.3$ & $5.8 \pm 0.7$ & $24.6 \pm 1.9$ & 1.37 & $\mathrm{~N}$ \\
\hline Arp299-B & 44.9 & 0.01041 & $\ldots$ & $1.6 \pm 0.3$ & $7.3 \pm 2.1$ & $18.8 \pm 2.2$ & $26.7 \pm 2.5$ & $27.2 \pm 7.9$ & $3.8 \pm 0.4$ & $21.8 \pm 2.1$ & 1.29 & $\mathrm{~N}$ \\
\hline Arp299-C & 44.9 & 0.01041 & $\ldots$ & $1.4 \pm 0.3$ & $6.8 \pm 1.6$ & $17.7 \pm 2.1$ & $21.8 \pm 2.2$ & $25.2 \pm 6.0$ & $3.6 \pm 0.4$ & $17.8 \pm 1.8$ & 1.26 & $\mathrm{~N}$ \\
\hline CGCG049-057 & 56.2 & 0.01300 & 9.8 & $1.8 \pm 0.4$ & $2.3 \pm 0.7$ & $\ldots$ & $\ldots$ & $5.5 \pm 1.6$ & $\ldots$ & $\ldots$ & $\ldots$ & $\mathrm{N}$ \\
\hline ESO173-G015 & 42.0 & 0.00974 & 10.4 & $3.7 \pm 0.7$ & $7.1 \pm 1.5$ & $\ldots$ & $\ldots$ & $30.1 \pm 6.4$ & $\ldots$ & $\ldots$ & $\ldots$ & $\mathrm{N}$ \\
\hline IRASF18293-3413 & 78.9 & 0.01818 & 10.9 & $6.1 \pm 1.2$ & $20.9 \pm 2.6$ & $118.6 \pm 11.0$ & $78.9 \pm 8.0$ & $25.4 \pm 3.2$ & $7.9 \pm 0.7$ & $21.1 \pm 2.1$ & 1.10 & $\mathrm{~N}$ \\
\hline M82 & 3.4 & 0.00094 & 10.0 & $0.4 \pm 0.1$ & $0.5 \pm 0.1$ & $4.0 \pm 0.3$ & $3.8 \pm 0.3$ & $352.8 \pm 53.5$ & $140.5 \pm 9.9$ & $537.2 \pm 41.2$ & 2.08 & $\mathrm{~N}$ \\
\hline MCG+12-02-001 & 68.0 & 0.01570 & $\cdots$ & $2.6 \pm 0.5$ & $4.1 \pm 1.1$ & $49.0 \pm 9.8$ & $52.9 \pm 8.0$ & $6.7 \pm 1.8$ & $4.4 \pm 0.9$ & $19.0 \pm 2.9$ & 1.53 & $\mathrm{~N}$ \\
\hline Mrk331 & 80.3 & 0.01848 & 10.7 & $3.0 \pm 0.6$ & $7.3 \pm 1.6$ & $31.1 \pm 5.4$ & $22.3 \pm 3.4$ & $8.6 \pm 1.9$ & $2.0 \pm 0.3$ & $5.8 \pm 0.9$ & 1 & $\mathrm{~N}$ \\
\hline NGC0253 & 3.4 & 0.00081 & 10.4 & $0.3 \pm 0.1$ & $0.9 \pm 0.1$ & $5.5 \pm 0.4$ & $5.1 \pm 0.4$ & $570.8 \pm 76.2$ & $191.6 \pm 13.3$ & $711.1 \pm 52.8$ & 2.19 & $\mathrm{Y}$ \\
\hline NGC1068 & 16.1 & 0.00379 & 11.0 & $1.3 \pm 0.3$ & $5.6 \pm 0.4$ & $36.2 \pm 4.7$ & $33.8 \pm 3.2$ & $161.0 \pm 11.8$ & $57.3 \pm 7.5$ & $214.1 \pm 20.3$ & 1.87 & $\mathrm{Y}$ \\
\hline NGC1365-NE & 23.1 & 0.00546 & $\cdots$ & $1.4 \pm 0.3$ & $5.3 \pm 0.6$ & $48.0 \pm 1.2$ & $24.1 \pm 3.9$ & $73.8 \pm 8.6$ & $37.0 \pm 0.9$ & $74.1 \pm 12.0$ & 1.76 & Y \\
\hline NGC1365-SW & 23.1 & 0.00546 & $\cdots$ & $1.4 \pm 0.3$ & $7.3 \pm 0.7$ & $74.3 \pm 1.7$ & $29.6 \pm 4.8$ & $103.1 \pm 10.5$ & $57.3 \pm 1.3$ & $91.2 \pm 14.8$ & 2.79 & $\mathrm{Y}$ \\
\hline NGC3256 & 40.4 & 0.00935 & 10.8 & $4.2 \pm 0.8$ & $9.2 \pm 1.1$ & $56.6 \pm 5.4$ & $128.9 \pm 13.3$ & $42.7 \pm 4.9$ & $14.3 \pm 1.4$ & $130.7 \pm 13.5$ & 1.29 & $\mathrm{~N}$ \\
\hline NGC5135 & 59.3 & 0.01369 & 10.9 & $1.8 \pm 0.4$ & $9.3 \pm 1.1$ & $40.6 \pm 3.7$ & $\ldots$ & $20.0 \pm 2.3$ & $4.8 \pm 0.4$ & $\ldots$ & 1.11 & $\mathrm{Y}$ \\
\hline NGC6240 & 106.8 & 0.02448 & 11.4 & $6.8 \pm 1.4$ & $25.3 \pm 5.0$ & $79.3 \pm 8.1$ & $149.1 \pm 29.9$ & $16.9 \pm 3.4$ & $2.9 \pm 0.3$ & $21.9 \pm 4.4$ & 1 & $\mathrm{Y}$ \\
\hline NGC7469 & 70.8 & 0.01632 & 11.1 & $3.5 \pm 0.7$ & $10.5 \pm 1.8$ & $41.4 \pm 3.4$ & $101.3 \pm 15.6$ & $15.9 \pm 2.7$ & $3.4 \pm 0.3$ & $33.6 \pm 5.2$ & 1.22 & $\mathrm{Y}$ \\
\hline NGC7552 & 22.8 & 0.00536 & 10.5 & $1.0 \pm 0.2$ & $2.6 \pm 0.4$ & $13.0 \pm 0.9$ & $17.2 \pm 1.7$ & $37.8 \pm 6.3$ & $10.3 \pm 0.8$ & $54.5 \pm 5.5$ & 1.45 & $\mathrm{~N}$ \\
\hline NGC7582 & 22.3 & 0.00525 & 10.6 & $0.7 \pm 0.1$ & $2.7 \pm 0.4$ & $14.7 \pm 1.1$ & $21.6 \pm 2.3$ & $41.2 \pm 6.1$ & $12.2 \pm 0.9$ & $71.4 \pm 7.6$ & 1.87 & $\mathrm{Y}$ \\
\hline NGC7771 & 61.8 & 0.01427 & 11.2 & $2.4 \pm 0.5$ & $8.5 \pm 1.1$ & $67.9 \pm 5.3$ & $77.9 \pm 11.7$ & $16.8 \pm 2.1$ & $7.4 \pm 0.6$ & $33.9 \pm 5.1$ & 1.68 & $\mathrm{~N}$ \\
\hline VV340A & 147.9 & 0.03367 & 11.2 & $5.3 \pm 1.1$ & $33.6 \pm 8.9$ & $108.6 \pm 17.4$ & $\ldots$ & $11.8 \pm 3.1$ & $2.1 \pm 0.3$ & $\ldots$ & 1.08 & $\mathrm{~N}$ \\
\hline CenA & 7.7 & 0.00183 & 11.1 & $0.4 \pm 0.1$ & $3.1 \pm 0.2$ & $21.0 \pm 2.1$ & $10.6 \pm 1.5$ & $392.1 \pm 26.2$ & $144.5 \pm 14.8$ & $291.1 \pm 41.2$ & 9.80 & $\mathrm{Y}$ \\
\hline IC1623 & 87.3 & 0.02007 & $\ldots$ & $4.7 \pm 0.9$ & $15.4 \pm 2.7$ & $52.6 \pm 5.8$ & $77.6 \pm 11.8$ & $15.4 \pm 2.7$ & $2.9 \pm 0.3$ & $17.0 \pm 2.6$ & 1 & $\mathrm{~N}$ \\
\hline NGC0034 & 85.3 & 0.01962 & 10.7 & $3.2 \pm 0.6$ & $5.1 \pm 1.5$ & $24.9 \pm 2.4$ & $13.8 \pm 2.8$ & $5.4 \pm 1.5$ & $1.4 \pm 0.1$ & $3.2 \pm 0.7$ & 1.07 & $\mathrm{Y}$ \\
\hline NGC0891-North & 7.4 & 0.00176 & $\ldots$ & $0.14 \pm 0.03$ & $0.8 \pm 0.2$ & $6.4 \pm 1.0$ & $6.9 \pm 1.0$ & $107.5 \pm 20.7$ & $47.9 \pm 7.3$ & $207.8 \pm 31.2$ & 4.88 & $\mathrm{~N}$ \\
\hline NGC0891-South & 7.4 & 0.00176 & $\ldots$ & $0.13 \pm 0.03$ & $1.7 \pm 0.3$ & $\ldots$ & $\ldots$ & $230.4 \pm 42.3$ & $\ldots$ & $\ldots$ & $\ldots$ & $\mathrm{N}$ \\
\hline NGC2146-NUC & 12.6 & 0.00298 & $\ldots$ & $0.6 \pm 0.1$ & $0.9 \pm 0.1$ & $7.1 \pm 0.2$ & $7.7 \pm 0.7$ & $42.9 \pm 6.8$ & $18.4 \pm 0.6$ & $79.3 \pm 7.2$ & 1.65 & $\mathrm{~N}$ \\
\hline NGC2146-NW & 12.6 & 0.00298 & $\ldots$ & $0.6 \pm 0.1$ & $1.2 \pm 0.2$ & $7.5 \pm 0.2$ & $8.9 \pm 0.8$ & $55.8 \pm 7.1$ & $19.4 \pm 0.6$ & $91.4 \pm 8.4$ & 1.69 & $\mathrm{~N}$ \\
\hline NGC2146-SE & 12.6 & 0.00298 & $\ldots$ & $0.6 \pm 0.1$ & $1.1 \pm 0.1$ & $7.8 \pm 0.2$ & $8.1 \pm 1.6$ & $49.4 \pm 6.9$ & $20.2 \pm 0.6$ & $83.8 \pm 16.8$ & 1.76 & $\mathrm{~N}$ \\
\hline NGC3227 & 16.3 & 0.00386 & 10.2 & $0.07 \pm 0.01$ & $0.9 \pm 0.2$ & $5.8 \pm 0.6$ & $\cdots$ & $25.0 \pm 6.7$ & $9.0 \pm 1.0$ & $\ldots$ & 2.27 & $\mathrm{Y}$ \\
\hline
\end{tabular}

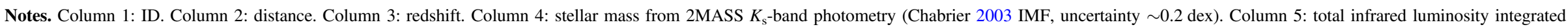

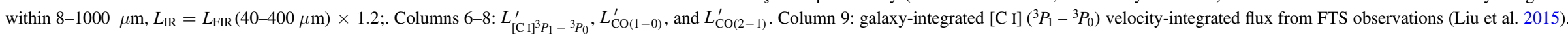

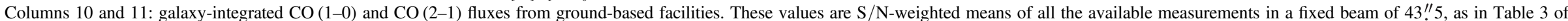

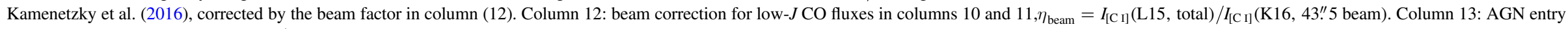
in Véron-Cetty \& Véron 2010: Y(es)/N(o).

References. Columns 5 and 9: Liu et al. (2015), this work; columns 10 and 11: Kamenetzky et al. (2016); column 13: Véron-Cetty \& Véron (2010).

(This table is available in its entirety in FITS format.) 
Table 4

Local Galaxies

\begin{tabular}{|c|c|c|c|}
\hline (1) & $\begin{array}{c}M_{[\mathrm{C} \mathrm{I}]} \\
\left(10^{5} M_{\odot}\right) \\
(2)\end{array}$ & $\begin{array}{c}M_{\text {gas }}(\mathrm{CO}) \\
\left(10^{9} M_{\odot}\right) \\
(3)\end{array}$ & $\begin{array}{c}{[\mathrm{C} \mathrm{I}] /\left[\mathrm{H}_{2}\right]_{(\mathrm{CO})}} \\
\left(\times 10^{-5}\right) \\
(4)\end{array}$ \\
\hline Arp193 & $13.0 \pm 4.1$ & $3.2 \pm 1.5$ & $9.3 \pm 5.3$ \\
\hline Arp220 & $17.3 \pm 3.5$ & $6.5 \pm 3.0$ & $6.0 \pm 3.1$ \\
\hline Arp299-A & $6.0 \pm 1.5$ & $2.3 \pm 1.1$ & $6.0 \pm 3.2$ \\
\hline Arp299-B & $9.1 \pm 2.7$ & $1.5 \pm 0.7$ & $13.7 \pm 7.7$ \\
\hline Arp299-C & $8.4 \pm 2.0$ & $1.4 \pm 0.7$ & $13.5 \pm 7.2$ \\
\hline CGCG049-057 & $2.9 \pm 0.8$ & $\ldots$ & $\ldots$ \\
\hline ESO173-G015 & $8.8 \pm 1.9$ & $\ldots$ & $\ldots$ \\
\hline IRASF18293-3413 & $26.0 \pm 3.3$ & $9.5 \pm 4.5$ & $6.2 \pm 3.0$ \\
\hline M82 & $0.7 \pm 0.1$ & $0.3 \pm 0.1$ & $4.9 \pm 2.4$ \\
\hline $\mathrm{MCG}+12-02-001$ & $5.1 \pm 1.3$ & $3.9 \pm 2.0$ & $3.0 \pm 1.7$ \\
\hline Mrk331 & $9.2 \pm 2.0$ & $2.5 \pm 1.2$ & $8.4 \pm 4.5$ \\
\hline NGC0253 & $1.1 \pm 0.1$ & $0.4 \pm 0.2$ & $5.8 \pm 2.8$ \\
\hline NGC1068 & $7.0 \pm 0.5$ & $2.9 \pm 1.4$ & $5.4 \pm 2.6$ \\
\hline NGC1365-NE & $6.6 \pm 0.8$ & $3.8 \pm 1.8$ & $3.9 \pm 1.8$ \\
\hline NGC1365-SW & $9.2 \pm 0.9$ & $5.9 \pm 2.7$ & $3.5 \pm 1.7$ \\
\hline NGC3256 & $11.5 \pm 1.3$ & $4.5 \pm 2.1$ & $5.8 \pm 2.8$ \\
\hline NGC5135 & $11.6 \pm 1.3$ & $3.2 \pm 1.5$ & $8.1 \pm 3.9$ \\
\hline NGC6240 & $31.6 \pm 6.3$ & $6.3 \pm 3.0$ & $11.3 \pm 5.8$ \\
\hline NGC7469 & $13.1 \pm 2.3$ & $3.3 \pm 1.5$ & $9.0 \pm 4.5$ \\
\hline NGC7552 & $3.3 \pm 0.5$ & $1.0 \pm 0.5$ & $7.1 \pm 3.5$ \\
\hline NGC7582 & $3.4 \pm 0.5$ & $1.2 \pm 0.6$ & $6.6 \pm 3.2$ \\
\hline NGC7771 & $10.6 \pm 1.3$ & $5.4 \pm 2.5$ & $4.4 \pm 2.1$ \\
\hline VV340A & $42.0 \pm 11.1$ & $8.7 \pm 4.2$ & $10.9 \pm 6.1$ \\
\hline CenA & $3.9 \pm 0.3$ & $1.7 \pm 0.8$ & $5.3 \pm 2.5$ \\
\hline IC1623 & $19.2 \pm 3.3$ & $4.2 \pm 2.0$ & $10.4 \pm 5.2$ \\
\hline NGC0034 & $6.4 \pm 1.8$ & $2.0 \pm 0.9$ & $7.3 \pm 4.0$ \\
\hline NGC0891-North & $1.0 \pm 0.2$ & $0.5 \pm 0.2$ & $4.4 \pm 2.3$ \\
\hline NGC0891-South & $2.1 \pm 0.4$ & $\ldots$ & $\ldots$ \\
\hline NGC2146-NUC & $1.1 \pm 0.2$ & $0.6 \pm 0.3$ & $4.5 \pm 2.2$ \\
\hline NGC2146-NW & $1.5 \pm 0.2$ & $0.6 \pm 0.3$ & $5.6 \pm 2.7$ \\
\hline NGC2146-SE & $1.3 \pm 0.2$ & $0.6 \pm 0.3$ & $4.7 \pm 2.3$ \\
\hline NGC3227 & $1.1 \pm 0.3$ & $0.5 \pm 0.2$ & $5.4 \pm 2.9$ \\
\hline
\end{tabular}

Note. Column 1: ID. Column 2: mass of [C I] (from $L_{[\mathrm{C} \mathrm{I}]^{3} P_{1}-{ }^{3} P_{0}}$ assuming $\left.T_{\mathrm{ex}}=30 \mathrm{~K}\right)$. Column 3: gas mass from $\mathrm{CO}(1-0)$, assuming $\alpha_{\mathrm{CO}}=0.8$ $M_{\odot} /\left(\mathrm{K} \mathrm{km} \mathrm{s}^{-1} \mathrm{pc}^{2}\right)$ (with an uncertainty of $0.2 \mathrm{dex}$ ). Column 4: atomic carbon abundance adopting $M_{\mathrm{gas}}(\mathrm{CO})$ and removing the $1.36 \times$ contribution of helium. (This table is available in its entirety in FITS format.)

We report the observed $[\mathrm{CI}]\left({ }^{3} P_{1}-{ }^{3} P_{0}\right)$ and $\mathrm{CO}(2-1)$ fluxes and $L^{\prime}$ luminosities in Table 1 . A full compilation, including the $\mathrm{CO}(4-3)$ and $\mathrm{CO}(5-4)$ fluxes, will be discussed in future work (F. Valentino et al. 2018,in preparation).

\section{Literature Data}

We compared our results with observations available in the literature. We collected and homogenized line luminosities and galaxy properties to properly match our conventions.

\subsection{Local Galaxies}

This sample is drawn from the public compilation of all Herschel/Fourier Transform Spectrometer (FTS) observations in the Herschel Science Archive of local galaxies by Liu et al. (2015, L15 hereafter). These sources are part of the IRAS Revised Bright Galaxy Sample (Sanders et al. 2003) and covered at $70-160 \mu \mathrm{m}$ by Herschel/PACS. The FTS simultaneously spanned the $446-1543 \mathrm{GHz}$ frequency interval, covering all CO lines with $J_{\text {up }}=4-13$, the [C I] $\left({ }^{3} P_{1}-{ }^{3} P_{0}\right)$ and [C I] $\left.{ }^{3} P_{2}-{ }^{3} P_{1}\right)$ lines, and several other transitions. L15 reduced the FTS raw data with the SPIRE v.12 calibration products and the Herschel Interactive Processing Environment pipelines (HIPE v.12.1.0; Ott 2010). They extracted all the lines simultaneously with customized optimized HIPE spectral-line-fitting scripts on the unapodized spectra with varied-width Sinc-convolved Gaussian functions, and they estimated the line flux errors from the rms of the spectra near each line (see L15 for further details). In total, we retrieved 32 galaxies (out of 146 in the compilation by L15) with a [C I] $\left({ }^{3} P_{1}-{ }^{3} P_{0}\right)>3 \sigma$ detection up to $z \sim 0.03$. We corrected the far-infrared luminosities integrated over the 40-400 $\mu \mathrm{m}$ interval reported in L15 to match the 8-1000 $\mu \mathrm{m}$ total $L_{\mathrm{IR}}$ we adopt here by multiplying by a factor of $1.2 \times$. We obtained this value by comparing the original $L_{\mathrm{FIR}}(40-400 \mu \mathrm{m})$ with $L_{\mathrm{IR}}(8-1000 \mu \mathrm{m})$ from SED modeling as described in Section 2.2 for the subset of galaxies from the Great Observatories All-Sky LIRGs Survey (Armus et al. 2009) included in L15. Emission-line fluxes and infrared luminosities have been beam-matched as described in L15 and reported to the total, galaxy-integrated values by applying the beam correction based on PACS photometry $\left(I_{\left[C_{1}\right]}(\mathrm{L} 15\right.$, total $)=$ $I_{\text {[C I] }}(\mathrm{L} 15$, beam $\left.) \times F_{\mathrm{PACS}, \text { total }} / F_{\mathrm{PACS}, \text { beam }}\right)$.

We further cross-matched the sample in L15 with the alternative compilation of Herschel/FTS observations and low-J CO transitions from ground-based facilities by Kamenetzky et al. (2016, K16). First, we checked that the FTS beam measurements for galaxies with $[\mathrm{CI}]\left({ }^{3} P_{1}-{ }^{3} P_{0}\right)$ detections in both samples were consistent. Then, we corrected the $\mathrm{CO}$ line measurements within the fixed $43 . " 5$ beam in K16 (their Table 3) to the galaxyintegrated values. We multiplied the fluxes in $\mathrm{K} 16$ by $\eta_{\text {beam }}=$ $I_{[\mathrm{C}]}(\mathrm{L} 15$, total $) / I_{[\mathrm{C} \mathrm{I}]}(\mathrm{K} 16,43$." 5 beam $)$, where $I_{[\mathrm{C} \mathrm{I}}(\mathrm{L} 15$, total $)$ are the galaxy-integrated [CI] $\left({ }^{3} P_{1}-{ }^{3} P_{0}\right)$ fluxes in $\mathrm{L} 15$ and $I_{[\mathrm{C} I]}(\mathrm{K} 16,43$ !' 5 beam $)$ are the $[\mathrm{C} \mathrm{I}]\left({ }^{3} P_{1}-{ }^{3} P_{0}\right)$ fluxes within a fixed 43 ." 5 beam by K16. The median correction factor is $2 \times$ and $1.25 \times$ for sources closer and farther than $20 \mathrm{Mpc}$, respectively. When multiple estimates of the same low- $J$ CO transitions were available, we assumed an $\mathrm{S} / \mathrm{N}$-weighted average as representative of the line flux. Note that the line ratios do not suffer from extra uncertainty due to the beam correction than what is reported in the K16 compilation. Moreover, for the closest sources, the final values might be representative mainly of the nuclear regions. Out of 32 sources with a [C I] $\left({ }^{3} P_{1}-{ }^{3} P_{0}\right)$ detection, 29 and 26 have a $\mathrm{CO}(1-0)$ and $\mathrm{CO}(2-1)>3 \sigma$ detection, respectively.

We further estimated the stellar masses of 20/32 galaxies of the FTS sample with available $K_{\mathrm{s}}$-band imaging from 2MASS (Skrutskie et al. 2006), averaging the values obtained following Arnouts et al. (2007, Equation (2)) and Juneau et al. (2011, Equation (B1), (B2)). We checked these results against the full UV-to-near-infrared SED fitting for a subset of 32 objects in common between the whole compilation of L15 and the sample studied by $\mathrm{U}$ et al. (2012), finding consistent results. Figure 1 shows the location of the galaxies with a $>3 \sigma[\mathrm{C} \mathrm{I}]\left({ }^{3} P_{1}-{ }^{3} P_{0}\right)$ detection in the $M_{\star}-\operatorname{SFR}\left(L_{\mathrm{IR}}\right)$ plane, typically lying above the main sequence at their redshift. Since AGNs can contaminate both $L_{\mathrm{IR}}$ and the $K_{\mathrm{s}}$-band derived $M_{\star}$, we flagged 12/32 known active galaxies listed in the catalog by Véron-Cetty \& Véron (2010, VCV10). Among the nonactive galaxies, $70 \%(14 / 20)$ are luminous infrared galaxies (LIRGs) $\left(L_{\mathrm{IR}}>10^{11} L_{\odot}\right)$. For the local sample, we do not attempt to disentangle the contribution of the dusty torus to the total $L_{\mathrm{IR}}$. In the rest of the paper, we will refer to the sample of local galaxies as "FTS local" or "local LIRGs" when they do not host a bright AGN. 
Table 5

SMGs and QSOs at $z \gtrsim 2.5$

\begin{tabular}{|c|c|c|c|c|c|c|c|c|c|c|c|c|c|c|}
\hline (1) & $\begin{array}{l}z_{\text {spec }} \\
\text { (2) }\end{array}$ & $\begin{array}{l}\mu_{\text {grav }} \\
\text { (3) }\end{array}$ & $\begin{array}{c}L_{\mathrm{IR}} \\
\left(10^{12} L_{\odot}\right) \\
(4)\end{array}$ & $\begin{array}{c}L_{\mathrm{FIR}} \\
\left(10^{12} L_{\odot}\right) \\
(5)\end{array}$ & $\begin{array}{c}L_{[\mathrm{CI}]^{3} P_{1}-{ }^{3} P_{0}} \\
\left(10^{10} \mathrm{~K} \mathrm{~km} \mathrm{~s}^{-1} \mathrm{pc}^{2}\right) \\
(6)\end{array}$ & $\begin{array}{c}L_{\mathrm{CO}(3-2)}^{\prime} \\
\left(10^{10} \mathrm{~K} \mathrm{~km} \mathrm{~s}^{-1} \mathrm{pc}^{2}\right) \\
(7)\end{array}$ & $\begin{array}{c}I_{[\mathrm{CI}]^{3} P_{1}-{ }^{3} P_{0}} \\
\left(\mathrm{Jy} \mathrm{km} \mathrm{s}{ }^{-1}\right) \\
(8)\end{array}$ & $\begin{array}{c}I_{\mathrm{CO}(3-2)} \\
\left(\mathrm{Jy} \mathrm{km} \mathrm{s}^{-1}\right) \\
(9)\end{array}$ & $\begin{array}{c}M_{\text {dust }} \\
\left(10^{8} M_{\odot}\right) \\
(10)\end{array}$ & $\begin{array}{c}M_{[\mathrm{C}]]} \\
\left(10^{6} M_{\odot}\right) \\
(11)\end{array}$ & $\begin{array}{c}\frac{[\mathrm{Ci}]}{\left.\left[\mathrm{C}_{2}\right]\right]_{\text {dust }}} \\
\left(\times 10^{-5}\right) \\
(12)\end{array}$ & $\begin{array}{c}\frac{[\mathrm{C}]}{\left[\mathrm{H}_{2}\right] \mathrm{CO}} \\
\left(\times 10^{-5}\right. \\
(13)\end{array}$ & $\begin{array}{l}f_{\mathrm{AGN}} \\
(14)\end{array}$ & $\begin{array}{l}\text { QSOs } \\
\text { (15) }\end{array}$ \\
\hline SMMJ02399-0136 & 2.808 & 2.5 & $9.2 \pm 0.9$ & $6.1 \pm 0.6$ & $1.48 \pm 0.16$ & $4.88 \pm 0.63$ & $1.9 \pm 0.2$ & $3.1 \pm 0.4$ & $32.3 \pm 1.1$ & $18.4 \pm 1.9$ & $4.3 \pm 2.0$ & $5.6 \pm 2.7$ & 0.09 & $\mathrm{~N}$ \\
\hline APM08279+5255 & 3.911 & 80 & $16.0 \pm 0.4$ & $3.6 \pm 0.1$ & $0.04 \pm 0.01$ & $\ldots$ & $0.9 \pm 0.1$ & $\ldots$ & $\ldots$ & $0.5 \pm 0.1$ & $\ldots$ & $\ldots$ & 0.99 & $\mathrm{Y}$ \\
\hline RXJ0911+0551 & 2.796 & 20 & $10.6 \pm 1.6$ & $2.5 \pm 0.4$ & $0.20 \pm 0.03$ & $0.39 \pm 0.06$ & $2.1 \pm 0.3$ & $2.0 \pm 0.3$ & $4.7 \pm 0.7$ & $2.5 \pm 0.4$ & $4.0 \pm 2.0$ & $9.5 \pm 4.8$ & 0.98 & Y \\
\hline F10214 & 2.285 & 10 & $33.0 \pm 1.5$ & $10.1 \pm 0.5$ & $0.27 \pm 0.05$ & $1.16 \pm 0.22$ & $2.0 \pm 0.4$ & $4.2 \pm 0.8$ & $6.6 \pm 0.5$ & $3.4 \pm 0.7$ & $3.9 \pm 2.0$ & $4.3 \pm 2.3$ & 0.74 & Y \\
\hline SMMJ123549+6215 & 2.202 & 1 & $4.2 \pm 1.3$ & $3.6 \pm 1.1$ & $1.41 \pm 0.26$ & $4.15 \pm 0.52$ & $1.1 \pm 0.2$ & $1.6 \pm 0.2$ & $64.6 \pm 20.1$ & $17.6 \pm 3.2$ & $2.1 \pm 1.2$ & $6.2 \pm 3.2$ & $\cdots$ & $\mathrm{N}$ \\
\hline BRI1335-0417 & 4.407 & 1 & $49.6 \pm 3.8$ & $20.8 \pm 1.6$ & $<8.83$ & $\ldots$ & $<2.2$ & $\ldots$ & $59.8 \pm 4.6$ & $<110.2$ & $<13.9$ & $\ldots$ & 0.29 & Y \\
\hline SMMJ14011+0252 & 2.565 & $4^{\mathrm{a}}$ & $3.7 \pm 0.1$ & $2.9 \pm 0.1$ & $0.75 \pm 0.12$ & $2.36 \pm 0.25$ & $1.8 \pm 0.3$ & $2.8 \pm 0.3$ & $7.12 \pm 0.02$ & $9.4 \pm 1.6$ & $9.9 \pm 4.9$ & $5.8 \pm 2.9$ & 0.05 & $\mathrm{~N}$ \\
\hline Cloverleaf & 2.558 & 11 & $26.9 \pm 0.6$ & $6.3 \pm 0.1$ & $0.59 \pm 0.09$ & $4.03 \pm 0.06$ & $3.9 \pm 0.6$ & $13.2 \pm 0.2$ & $8.7 \pm 0.4$ & $7.3 \pm 1.1$ & $6.4 \pm 3.1$ & $2.7 \pm 1.3$ & 0.98 & $\mathrm{Y}$ \\
\hline SMMJ16359+6612 & 2.517 & $22 \pm 2$ & $0.74 \pm 0.03$ & $0.55 \pm 0.02$ & $0.12 \pm 0.02$ & $0.42 \pm 0.03$ & $1.7 \pm 0.3$ & $2.8 \pm 0.2$ & $4.60 \pm 0.04$ & $1.6 \pm 0.3$ & $2.6 \pm 1.3$ & $5.5 \pm 2.7$ & 0.12 & $\mathrm{~N}$ \\
\hline SMMJ163650+4057 & 2.385 & 1 & $8.4 \pm 0.4$ & $5.2 \pm 0.2$ & $<1.06$ & $4.77 \pm 0.60$ & $<0.7$ & $1.6 \pm 0.2$ & $38.4 \pm 1.8$ & $<13.2$ & $<2.6$ & $<4.1$ & $\cdots$ & $\mathrm{N}$ \\
\hline SMMJ163658+4105 & 2.452 & 1 & $9.5 \pm 0.7$ & $8.0 \pm 0.6$ & $1.39 \pm 0.31$ & $5.62 \pm 0.62$ & $0.9 \pm 0.2$ & $1.8 \pm 0.2$ & $22.9 \pm 1.7$ & $17.3 \pm 3.9$ & $5.7 \pm 2.9$ & $4.5 \pm 2.4$ & $\cdots$ & $\mathrm{N}$ \\
\hline MM18423+5938 & 3.930 & 20 & $10.2 \pm 1.0$ & $5.0 \pm 0.5$ & $0.39 \pm 0.08$ & $\ldots$ & $2.3 \pm 0.5$ & $\ldots$ & $7.88 \pm 0.04$ & $4.8 \pm 1.0$ & $4.6 \pm 2.4$ & $\ldots$ & 0.18 & $\mathrm{~N}$ \\
\hline SMMJ213511-0102 & 2.326 & $32.5 \pm 4.5$ & $2.4 \pm 0.1$ & $2.1 \pm 0.1$ & $0.69 \pm 0.02$ & $1.16 \pm 0.01$ & $16.0 \pm 0.5$ & $13.2 \pm 0.1$ & $8.7 \pm 0.2$ & $8.7 \pm 0.3$ & $7.5 \pm 3.5$ & $11.0 \pm 5.1$ & $\cdots$ & $\mathrm{N}$ \\
\hline PSSJ2322+1944 & 4.120 & $5.3 \pm 0.3$ & $17.8 \pm 1.7$ & $5.3 \pm 0.5$ & $0.55 \pm 0.08$ & $\ldots$ & $0.8 \pm 0.1$ & $\cdots$ & $14.5 \pm 1.4$ & $6.8 \pm 1.0$ & $3.6 \pm 1.8$ & $\ldots$ & 0.88 & $\mathrm{Y}$ \\
\hline ID141 & 4.243 & $20^{\mathrm{a}}$ & $5.4 \pm 0.3$ & $4.3 \pm 0.2$ & $0.53 \pm 0.17$ & $\ldots$ & $2.8 \pm 0.9$ & $\cdots$ & $7.9 \pm 0.4$ & $6.6 \pm 2.1$ & $6.3 \pm 3.5$ & $\ldots$ & $\ldots$ & $\mathrm{N}$ \\
\hline SXDF7 & 2.529 & 1 & $8.1 \pm 0.4$ & $6.0 \pm 0.3$ & $1.30 \pm 0.33$ & $\ldots$ & $0.8 \pm 0.2$ & $\ldots$ & $15.2 \pm 0.7$ & $16.2 \pm 4.1$ & $8.1 \pm 4.3$ & $\ldots$ & 0.01 & $\mathrm{~N}$ \\
\hline SXDF11 & 2.282 & 1 & $3.3 \pm 0.2$ & $2.4 \pm 0.1$ & $<0.95$ & $\ldots$ & $<0.7$ & $\ldots$ & $23.9 \pm 1.4$ & $<11.9$ & $<3.8$ & $\ldots$ & $\ldots$ & $\mathrm{N}$ \\
\hline SXDF4a $\mathrm{a}^{\mathrm{b}}$ & 2.030 & 1 & $4.6 \pm 0.2$ & $3.3 \pm 0.2$ & $0.78 \pm 0.22$ & $\ldots$ & $0.7 \pm 0.2$ & $\cdots$ & $21.5 \pm 1.1$ & $9.7 \pm 2.8$ & $3.4 \pm 1.9$ & $\ldots$ & $\ldots$ & $\mathrm{N}$ \\
\hline SXDF $4 b^{b}$ & 2.027 & 1 & $4.6 \pm 0.2$ & $3.3 \pm 0.2$ & $<0.77$ & $\ldots$ & $<0.7$ & $\ldots$ & $21.5 \pm 1.1$ & $<9.7$ & $<3.4$ & $\ldots$ & - & $\mathrm{N}$ \\
\hline SA22.96 & 2.517 & 1 & $7.6 \pm 0.3$ & $6.6 \pm 0.2$ & $1.13 \pm 0.32$ & $5.23 \pm 1.63$ & $0.7 \pm 0.2$ & $1.6 \pm 0.5$ & $26.9 \pm 1.0$ & $14.1 \pm 4.0$ & $4.0 \pm 2.2$ & $4.0 \pm 2.5$ & $\ldots$ & $\mathrm{N}$ \\
\hline
\end{tabular}

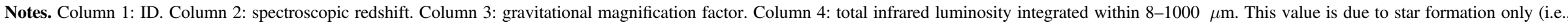

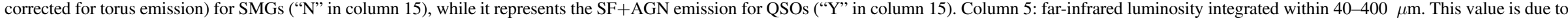

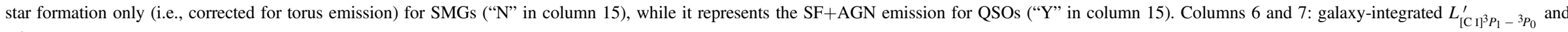

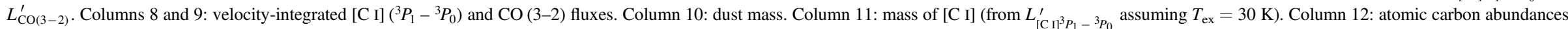

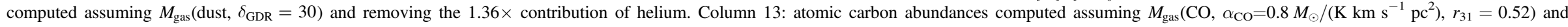

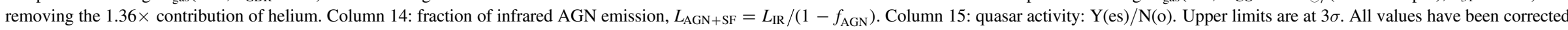
for gravitational magnification.

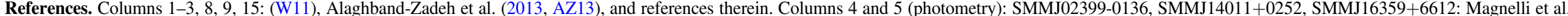

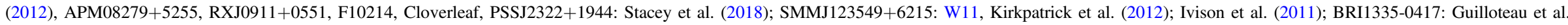

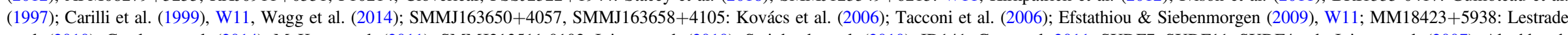

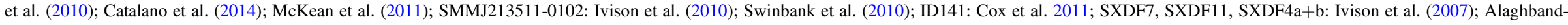
Zadeh et al. (2012, 2013); SA22.96: Menéndez-Delmestre et al. (2009); Alaghband-Zadeh et al. (2012, 2013).

${ }^{\mathrm{a}}$ From Alaghband-Zadeh et al. (2013).

${ }^{\mathrm{b}}$ This source is unresolved at $850 \mu \mathrm{m}$, but two components are evident in the $\mathrm{H} \alpha$ and $\mathrm{CO}$ (4-3) maps. The photometry refers to the unresolved source.

(This table is available in its entirety in FITS format.) 
Table 6

SPT SMGs at $z \sim 4$

\begin{tabular}{|c|c|c|c|c|c|c|c|c|c|c|c|c|c|c|}
\hline (1) & $\begin{array}{l}z_{\text {spec }} \\
\text { (2) }\end{array}$ & $\begin{array}{l}\mu_{\text {grav }} \\
\text { (3) }\end{array}$ & $\begin{array}{c}M_{\star} \\
\left(10^{10} M_{\odot}\right) \\
(4)\end{array}$ & $\begin{array}{c}L_{\mathrm{IR}} \\
\left(10^{12} L_{\odot}\right) \\
(5)\end{array}$ & $\begin{array}{c}L_{\mathrm{FIR}} \\
\left(10^{12} L_{\odot}\right) \\
(6)\end{array}$ & $\begin{array}{c}L_{[\mathrm{CI}]^{3} P_{1}-{ }^{3} P_{0}}^{\prime} \\
\left(10^{10} \mathrm{Km} \mathrm{s}^{-1} \mathrm{pc}^{2}\right) \\
(7)\end{array}$ & $\begin{array}{c}L_{\mathrm{CO}(2-1)}^{\prime} \\
\left(10^{10} \mathrm{~K} \mathrm{~km} \mathrm{~s}^{-1} \mathrm{pc}^{2}\right) \\
(8)\end{array}$ & $\begin{array}{c}I_{[\mathrm{C} \mathrm{I}]^{3} P_{1}-{ }^{3} P_{0}} \\
\left(\mathrm{Jy} \mathrm{km} \mathrm{s}{ }^{-1}\right) \\
(9)\end{array}$ & $\begin{array}{c}I_{\mathrm{CO}(2-1)} \\
\left(\mathrm{Jy} \mathrm{km} \mathrm{s}{ }^{-1}\right) \\
(10)\end{array}$ & $\begin{array}{c}M_{\text {dust }} \\
\left(10^{8} M_{\odot}\right) \\
(11)\end{array}$ & $\begin{array}{c}M_{[\mathrm{C}]} \\
\left(10^{6} M_{\odot}\right) \\
(12)\end{array}$ & $\begin{array}{c}\frac{[\mathrm{C}]]}{\left[\mathrm{H}_{2}\right] \mathrm{d} \text { dust }} \\
\left(\times 10^{-5}\right) \\
(13)\end{array}$ & $\begin{array}{c}\frac{[\mathrm{C}]]}{\left[\mathrm{H}_{2}\right] \mathrm{CO}} \\
\left(\times 10^{-5}\right) \\
(14)\end{array}$ & $f_{\mathrm{AGN}}$ \\
\hline SPT0113-46 & 4.233 & $23.9 \pm 0.5$ & $\ldots$ & $1.5 \pm 0.1$ & $1.2 \pm 0.1$ & $0.53 \pm 0.11$ & $1.22 \pm 0.09$ & $3.4 \pm 0.7$ & $1.7 \pm 0.1$ & $11.6 \pm 0.1$ & $6.6 \pm 1.3$ & $4.3 \pm 2.2$ & $12.9 \pm 6.6$ & $\ldots$ \\
\hline SPT0125-50 & 3.959 & $14.1 \pm 0.5$ & $\ldots$ & $10.4 \pm 0.5$ & $4.9 \pm 0.2$ & $0.57 \pm 0.13$ & $\ldots$ & $2.4 \pm 0.5$ & $\ldots$ & $19.0 \pm 2.0$ & $7.1 \pm 1.6$ & $2.8 \pm 1.5$ & $\ldots$ & 0.16 \\
\hline SPT0300-46 & 3.596 & $5.7 \pm 0.4$ & $\ldots$ & $8.8 \pm 0.2$ & $6.9 \pm 0.2$ & $<1.21$ & $\ldots$ & $1.8 \pm 0.8^{\mathrm{a}}$ & $\ldots$ & $27.3 \pm 2.0$ & $<15.1$ & $<4.2$ & $\ldots$ & $\ldots$ \\
\hline SPT0345-47 & 4.296 & $8.0 \pm 0.5$ & $\ldots$ & $36.0 \pm 1.7$ & $15.3 \pm 0.7$ & $<0.50$ & $3.95 \pm 0.44$ & $<1.0$ & $1.8 \pm 0.2$ & $17.0 \pm 0.9$ & $<6.2$ & $<2.7$ & $<3.7$ & $\cdots$ \\
\hline SPT0418-47 & 4.225 & $32.7 \pm 2.7$ & $\ldots$ & $3.0 \pm 0.1$ & $2.4 \pm 0.1$ & $0.28 \pm 0.07$ & $0.68 \pm 0.06$ & $2.5 \pm 0.6$ & $1.3 \pm 0.1$ & $4.4 \pm 0.1$ & $3.5 \pm 0.9$ & $6.1 \pm 3.2$ & $12.3 \pm 6.6$ & $\ldots$ \\
\hline SPT0441-46 & 4.477 & $12.7 \pm 1.0$ & $\ldots$ & $7.2 \pm 0.6$ & $3.9 \pm 0.4$ & $<0.72$ & $1.40 \pm 0.21$ & $1.8 \pm 0.7^{\mathrm{a}}$ & $0.9 \pm 0.1$ & $14.7 \pm 0.7$ & $<9.0$ & $<4.6$ & $<15.2$ & $\ldots$ \\
\hline SPT0459-59 & 4.799 & $3.6 \pm 0.3$ & $\cdots$ & $18.0 \pm 1.0$ & $9.8 \pm 0.5$ & $3.09 \pm 0.89$ & $6.37 \pm 0.46$ & $2.4 \pm 0.7$ & $1.1 \pm 0.1$ & $52.8 \pm 2.1$ & $38.5 \pm 11.1$ & $5.5 \pm 3.0$ & $14.4 \pm 7.9$ & $\cdots$ \\
\hline SPT0529-54 & 3.369 & $13.2 \pm 0.5$ & $\cdots$ & $3.5 \pm 0.1$ & $3.0 \pm 0.1$ & $0.57 \pm 0.11$ & $\ldots$ & $2.9 \pm 0.5$ & $\ldots$ & $33.3 \pm 0.8$ & $7.1 \pm 1.3$ & $1.6 \pm 0.8$ & $\ldots$ & $\cdots$ \\
\hline SPT0532-50 & 3.399 & $10.0 \pm 0.6$ & $\cdots$ & $7.6 \pm 0.7$ & $5.7 \pm 0.5$ & $0.85 \pm 0.20$ & $\ldots$ & $3.2 \pm 0.8$ & $\ldots$ & $57.4 \pm 1.3$ & $10.6 \pm 2.5$ & $1.4 \pm 0.7$ & $\ldots$ & 0.62 \\
\hline SPT2103-60 & 4.436 & $27.8 \pm 1.8$ & $\cdots$ & $1.80 \pm 0.03$ & $1.51 \pm 0.02$ & $0.45 \pm 0.11$ & $1.06 \pm 0.17$ & $3.1 \pm 0.8$ & $1.6 \pm 0.2$ & $6.4 \pm 0.1$ & $5.6 \pm 1.4$ & $6.6 \pm 3.4$ & $12.5 \pm 6.8$ & $\cdots$ \\
\hline SPT2132-58 & 4.768 & $5.7 \pm 0.5$ & $\ldots$ & $16.8 \pm 0.4$ & $7.7 \pm 0.2$ & $<0.69$ & $3.08 \pm 0.25$ & $0.8 \pm 0.3^{\mathrm{a}}$ & $0.8 \pm 0.1$ & $32.0 \pm 0.8$ & $<8.6$ & $<2.0$ & $<6.7$ & $\cdots$ \\
\hline SPT2146-55 & 4.567 & $6.7 \pm 0.4$ & $8.0_{-6.0}^{+19.0}$ & $11.4 \pm 0.4$ & $5.9 \pm 0.2$ & $1.73 \pm 0.45$ & $2.74 \pm 0.46$ & $2.7 \pm 0.7$ & $0.9 \pm 0.2$ & $20.9 \pm 1.5$ & $21.6 \pm 5.6$ & $7.8 \pm 4.2$ & $18.7 \pm 10.4$ & 0.13 \\
\hline SPT2147-50 & 3.760 & $6.6 \pm 0.4$ & $2.0_{-0.9}^{+1.8}$ & $7.5 \pm 1.1$ & $5.9 \pm 0.9$ & $0.95 \pm 0.28$ & $2.70 \pm 0.54$ & $2.0 \pm 0.6$ & $1.2 \pm 0.2$ & $19.5 \pm 0.5$ & $11.9 \pm 3.5$ & $4.6 \pm 2.5$ & $10.5 \pm 6.1$ & $\ldots$ \\
\hline
\end{tabular}

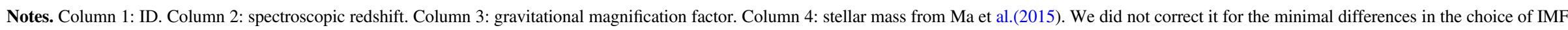

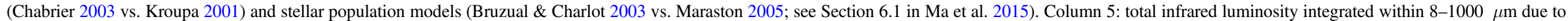

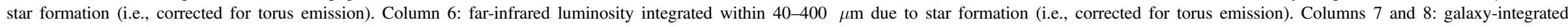

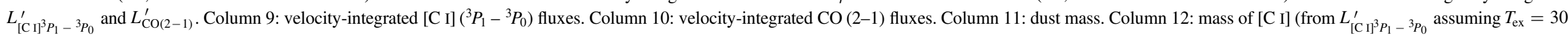

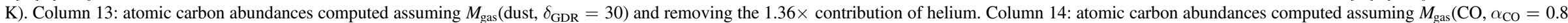

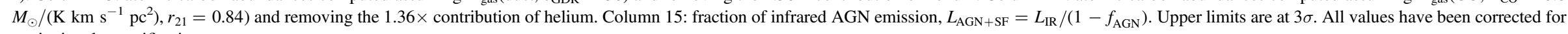
gravitational magnification.

${ }^{a}$ We report the nominal flux measurements presented in Bothwell et al. (2017), but we adopt the $3 \sigma$ upper limits for the analysis.

References. Columns 1-3, 9: Bothwell et al. (2017); column 4: Ma et al. (2015); columns 5 and 6 (photometry): Weiß et al. (2013); column 10: Aravena et al. (2016).

(This table is available in its entirety in FITS format.) 
All the physical quantities used in the analysis of the local galaxies are reported in Tables 3 and 4.

\subsection{High-redshift Submillimeter Galaxies and Quasars}

We assembled a sample of SMGs and quasars (QSOs) with [C I] $\left({ }^{3} P_{1}-{ }^{3} P_{0}\right)$ coverage from (W11), Alaghband-Zadeh et al. (2013, AZ13), and Bothwell et al. (2017, B17). We refer the reader to these papers for fully detailed references, sample selection, and observations. W11 and AZ13 targeted or collected information on typical SMGs at $z \sim 2.5-4$ detected at $\sim 850 \mu \mathrm{m}$, with a tail of well-studied QSOs extending up to $z \sim 6.5$. A large fraction of these sources are gravitationally magnified up to $\sim 30 \times$ and are detected in $\mathrm{CO}(3-2)$ and CO (4-3). Similarly magnified are $1.4 \mathrm{~mm}$ detected SMGs in B17, identified in a blank-field survey with the South Pole Telescope (SPT; Vieira et al. 2010; Weiß et al. 2013), with spectroscopic information on high- (Weiß et al. 2013, B17) and low- $J$ transitions $(\mathrm{CO}(2-1)$; Aravena et al. 2016). The final sample consist of 33 galaxies, $25 / 33$ with [C I] $\left({ }^{3} P_{1}-{ }^{3} P_{0}\right)$ detections at $>3 \sigma$. We rederived their total $L_{\mathrm{IR}}$ and dust masses $M_{\text {dust }}$, modeling their far-infrared SED by applying the same method described in Section 2.2. The SPT SMGs are all detected in the SPIRE 250, 350, and $500 \mu \mathrm{m}$; LABOCA $870 \mu \mathrm{m}$; SPT 1.4 and $2.0 \mathrm{~mm}$; and ALMA $3 \mathrm{~mm}$ bands (Weiß et al. 2013). The SEDs of SMGs and QSOs from W11 and AZ13 are sampled less homogeneously but ensuring good coverage of both the peak and the Rayleigh-Jeans of the dust emission in the vast majority of cases. Applying our recipes, we estimate a total $L_{\mathrm{IR}}(8-1000 \mu \mathrm{m}) \sim 1.5 \times(\sim 3 \times)$ larger than the original values derived with modified blackbody curves for SMGs (QSOs), including a correction for the different integration limits. We also estimate $M_{\text {dust }}$ for the SPT SMGs that is systematically larger than previously reported (Aravena et al. 2016). The systematic differences in $L_{\mathrm{IR}}$ and $M_{\text {dust }}$ fully depend on the adopted models (modified blackbody law; DL07) and their parameters (effective dust emissivity index $\beta$, dust mass absorption coefficient $\kappa$, peak temperature; Magdis et al. 2012b). The discrepancy in $L_{\mathrm{IR}}$ is larger for QSO hosts owing to the dusty torus emission mid-IR bands, where the difference between modified blackbody curves and DL07 models is more significant. All quantities presented here have been corrected for magnification. Moreover, we correct the $L_{\mathrm{IR}}$ luminosities of SMGs for the contribution of AGNs, similar to what we did for the MS sample. We find only one (SPT) SMG whose SED is dominated by a dusty torus $(\sim 70 \%$ of the total $\left.L_{\mathrm{IR}}\right)$. For known bright QSOs at high redshift in W11, we do not attempt to separate the star formation and AGN contributions to $L_{\mathrm{IR}}$, being largely dominated by the latter. However, we will not consider these sources in the analysis any further but simply show their position in the various plots for reference. Stellar masses are not available for this high-redshift sample, apart from two sources listed in B17. Therefore, we could not place these objects in the $M_{\star}$-SFR plane and canonically define them as starburst (SB) or MS based on these observables. However, their observed ISM conditions, gas and SFR densities, and star formation efficiencies (SFEs) generally distinguish SMGs from MS galaxies (e.g., Daddi et al. 2010a; Genzel et al. 2010; Bothwell et al. 2013; Casey et al. 2014). In the following, we will label the sample from W11 and AZ13 as "SMGs $(z \sim 2.5)$," the sources with clear AGN signatures from W11 as "QSOs (high-z)," and the sample by B17 as "SPT SMGs $(z \sim 4)$." Moreover, we will consider SMGs as starbursting systems and not typical MS galaxies. We report the main properties of these objects in Tables 5 and 6 .

\section{Analysis and Results}

$$
\text { 4.1. The } L_{I R}-L_{[C I]^{3} P_{1}-{ }^{3} P_{0}} \text { Relation }
$$

Following Solomon \& Vanden Bout (2005), we computed the [C I] $\left({ }^{3} P_{1}-{ }^{3} P_{0}\right)$ line luminosities in $\mathrm{K} \mathrm{km} \mathrm{s}^{-1} \mathrm{pc}^{2}$, representing the integrated source brightness temperature

$$
L_{\text {line }}^{\prime}\left[\mathrm{K} \mathrm{km} \mathrm{s}^{-1} \mathrm{pc}^{2}\right]=3.25 \times 10^{7} S_{\text {line }} \Delta v \nu_{\text {obs }}^{-2}(1+z)^{-3} D_{\mathrm{L}}^{2} \text {, }
$$

where $S_{\text {line }} \Delta v$ is the measured velocity-integrated line flux in $\mathrm{Jy} \mathrm{km} \mathrm{s}{ }^{-1}, \nu_{\mathrm{obs}}$ is the observed line frequency in $\mathrm{GHz}, z$ is the redshift of the source, and $D_{\mathrm{L}}$ is the luminosity distance in Mpc. Figure 2 shows the relation between the total $L_{\mathrm{IR}}$ and $L_{[\mathrm{C} I]^{3} P_{1}-{ }^{3} P_{0}}$ for our sample of MS galaxies and the literature compilation of starbursting sources at various redshifts. Both a Spearman's rank and a Pearson's correlation coefficient show that the two quantities are correlated, considering all $>3 \sigma$ detected sources and excluding QSOs and AGNs $\left(\rho_{\text {Spearman }}=0.8990, \rho_{\text {Pearson }}=0.9024\right)$. We further applied a linear regression analysis on $\left.\log \left(L_{\mathrm{IR}}\right)-\log \left(L_{[\mathrm{C}}^{\prime}\right]^{3} P_{1}-{ }^{3} P_{0}\right)$ using both a Bayesian (LINMIX_ERR.PRO; Kelly 2007) and a $\chi^{2}$-minimization algorithm (MPFIT.PRO; Markwardt 2009), taking into account the uncertainties on both $L_{\mathrm{IR}}$ and $L_{[\mathrm{C}]^{3} P_{1}-{ }^{3} P_{0}}^{\prime}$ and including the upper limits on $L_{[\mathrm{CI}]^{3} P_{1}-{ }^{3} P_{0}}^{\prime}$ in the Bayesian fit. Since $L_{\mathrm{IR}}$ and $L_{[\mathrm{C}]]^{3} P_{1}-{ }^{3} P_{0}}$ are proxies for the integrated SFR and $M_{\text {gas }}$, this relation is analogous to the Schmidt-Kennicutt relation (Schmidt 1959; Kennicutt 1998b; with the $X$ and $Y$ axes generally inverted). The two algorithms we applied provided fully consistent results within the uncertainties, and the effect of upper limits is negligible. We modeled a total of 57 [C I] $\left({ }^{3} P_{1}-{ }^{3} P_{0}\right)$-detected galaxies and 10 upper limits. The Bayesian best-fit model returns a sublinear slope of $0.78 \pm 0.05$ with an observed scatter of $\sigma=0.26$ dex. Note that AGNs and QSOs are not included in the fit or the calculation of $\sigma$. Their location in the diagram is mainly driven by their $L_{\mathrm{IR}}$, boosted by the contribution of the dusty tori in the mid-IR regime, adding to moderately larger intrinsic luminosities than high-redshift SMGs at fixed $L_{[\mathrm{C} I]^{3} P_{1}-{ }^{3} P_{0}}$ (W11). Modeling only the starbursting population (i.e., SB at $z \sim 1.2$, local LIRGs, and SMGs) provides a similar slope of $0.79 \pm 0.06$. Interestingly, these values are consistent with that of the $\log \left(L_{\mathrm{IR}}\right)-\log \left(L_{\mathrm{CO}(1-0)}^{\prime}\right)$ relation $(0.81 \pm 0.03$; Sargent et al. 2014), reinforcing the connection between [C I] and CO. Moreover, our MS-detected galaxies appear to have larger $L_{[\mathrm{C} I]^{3} P_{1}-{ }^{3} P_{0}}^{\prime} / L_{\mathrm{IR}}$ ratios than SMGs. This is more evident in the right panel of Figure 2. The mean value of

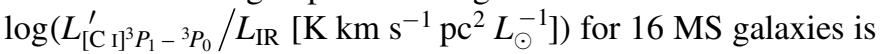
$(-2.43 \pm 0.06), \sim 2 \times$ higher than the mean for SMGs at $z \sim 2.5 \quad(-2.77 \pm 0.07$ dex $)$ and SPT SMGs at $z \sim 4$ $(-2.80 \pm 0.07 \mathrm{dex})$, where the uncertainties represent the error on the mean. We included the upper limits on $[\mathrm{CI}]\left({ }^{3} P_{1}-{ }^{3} P_{0}\right)$ in the calculation using a survival analysis (KM estimator; Kaplan \& Meier 1958). This difference is significant at an $\sim 4 \sigma$ level. The median values are fully consistent with the mean. 

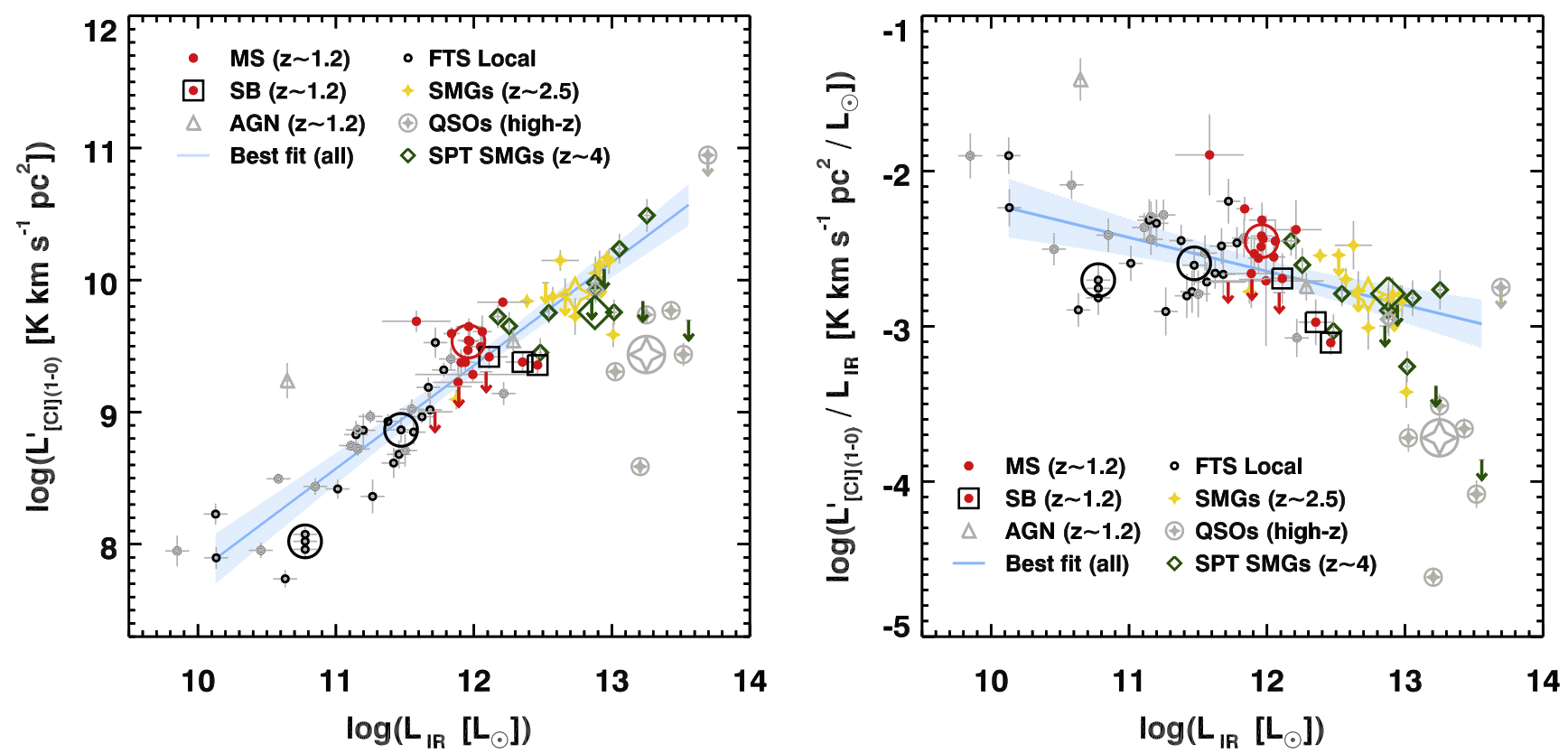

Figure 2. Total $L_{\mathrm{IR}}-L_{[\mathrm{C} \mathrm{I}]^{3} P_{1}-{ }^{3} P_{0}}$ relation. In both panels, red filled circles represent our $>3 \sigma$ detected MS galaxies at $z \sim 1.2$, open black squares represent SBs, gray triangles represent AGNs, open black circles represent local FTS $>3 \sigma$ detections without AGN signatures, open gray circles represent local FTS $>3 \sigma$ detections listed as active in VCV10, yellow stars represent SMGs at $z \sim 2.5$ from W11 and AZ13, gray stars and open circles represent high-redshift QSOs from W11, and open green

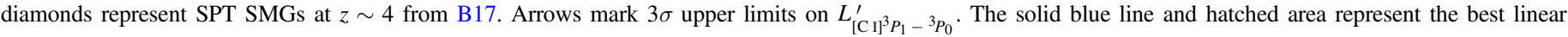
regression model of all SF-dominated galaxies (i.e., excluding QSOs and AGNs) and the 95\% confidence interval. The $L_{\mathrm{IR}}$ of the QSOs is dominated by the emission of the dusty torus, which is included in the estimate, at odds with the rest of the SF-dominated galaxies. The larger symbols mark the median values for each sample. We split the FTS local sample into two bins with a threshold at $L_{\mathrm{IR}}=10^{11} L_{\odot}$, the formal limit for the LIRG classification. Left: $L_{\mathrm{IR}}-L_{[\mathrm{C}]^{3} P_{1}-{ }^{3} P_{0}}$ relation, a proxy for the SFR and gas mass relation. Right: $L_{\mathrm{IR}}-L_{[\mathrm{C}]]^{3} P_{1}-{ }^{3} P_{0}} / L_{\mathrm{IR}}$ relation, where $L_{[\mathrm{C} \mathrm{I}]^{3} P_{1}-{ }^{3} P_{0}} / L_{\mathrm{IR}}$ is a proxy for the gas depletion timescale $\tau_{\mathrm{dep}} \propto 1 / \mathrm{SFE}$, dropping with increasing $L_{\mathrm{IR}}$ and shorter for starbursting galaxies than for sources on the MS.

The ratio for the local sample of nonactive galaxies is consistent with the estimate for MS galaxies, but it suffers from a very large dispersion. In Figure 3, we further show the $L_{[\mathrm{C} I]^{3} P_{1}-{ }^{3} P_{0}}^{\prime} / L_{\mathrm{IR}}$ ratio as a function of the distance from the main sequence $\left(\triangle \mathrm{SFR}=\mathrm{SFR} / \mathrm{SFR}_{\mathrm{MS}}\right)$ as parameterized in Sargent et al. (2014). We included only sources with a stellar mass estimate, i.e., all of our galaxies, part of the local LIRGs, and two SPT SMGs. Excluding galaxies with AGN signatures, the $L_{[\mathrm{C}]^{3} P_{1}-{ }^{3} P_{0}}^{\prime} / L_{\mathrm{IR}}$ ratio and $\Delta$ SFR are mildly anticorrelated $\left(\rho_{\text {Spearman }}=-0.6940, \rho_{\text {Pearson }}=-0.5961\right)$, with SMGs and SBs at $z \sim 1.2$ showing systematically lower ratios than MS galaxies, as in Figure 2. However, the scarce statistics of lower main-sequence sources and SBs with available $M_{\star}$ prevents us from deriving more definitive conclusions. From a physical perspective, since $L_{[\mathrm{C} I]^{3} P_{1}-{ }^{3} P_{0}}^{\prime}$ traces the gas mass and $L_{\mathrm{IR}}$ the SFR, their ratio is a proxy for the gas depletion timescale $\tau_{\text {dep }}$. The observed trends would then suggest a drop of this quantity (or, equivalently, an increment of SFE) with increasing $L_{\mathrm{IR}}$ and distance from the main sequence, analogous to the wellestablished correlations observed for CO (e.g., Daddi et al. 2010b; Magdis et al. 2012b; Genzel et al. 2015; Tacconi et al. 2018).

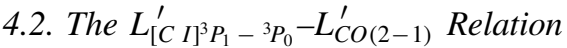

Figure 4 shows the $L_{[\mathrm{C}]^{3} P_{1}-{ }^{3} P_{0}}^{\prime} / L_{\mathrm{CO}(2-1)}^{\prime}$ ratio as a function of the total $L_{\mathrm{IR}}$. The observed ratio is similar in MS and SB galaxies

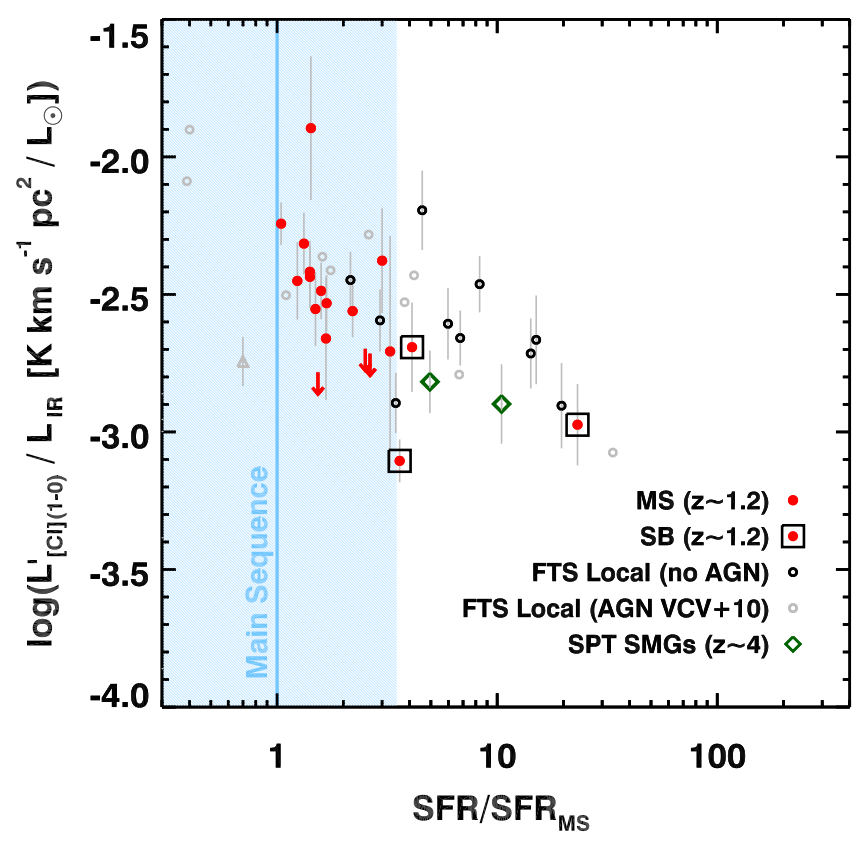

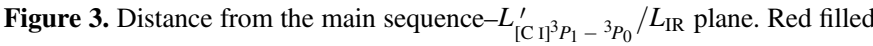
circles mark our sample of MS galaxies at $z \sim 1.2$ with a $>3 \sigma$ [C I $\left[{ }^{3} P_{1}-{ }^{3} P_{0}\right)$ detection. Black squares indicate SBs. Open black and gray circles represent the local comparison sample of nonactive and active galaxies covered by FTS observations (Liu et al. 2015). Open green diamonds indicate the two SPT SMGs at $z \sim 4$ with an estimate of $M_{\star}$ from B17. Arrows mark $3 \sigma$ upper limits on $L_{[\mathrm{C}]]^{3} P_{1}-{ }^{3} P_{0}}$. The MS is parameterized as in Sargent et al. (2014). 

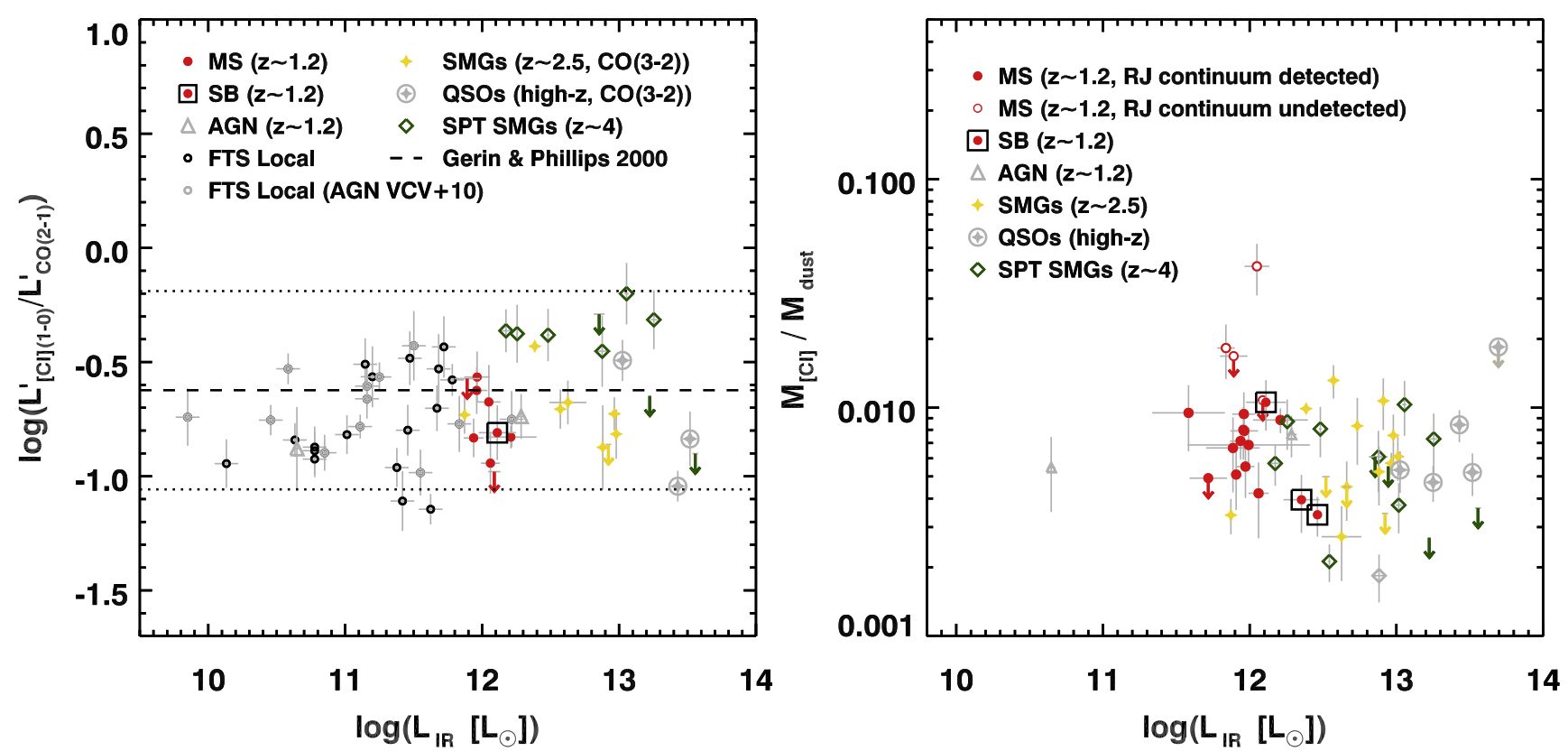

Figure 4. Left: $L_{[\mathrm{C}]]^{3} P_{1}-{ }^{3} P_{0}}^{\prime}-L_{\mathrm{CO}(2-1)}^{\prime}$ as a function of total $L_{\mathrm{IR}}$. Symbols are coded as in Figure 2 and reported in the legend. The dashed and dotted black lines mark the average ratio and its scatter observed in a sample of local spirals, mergers, and low-metallicity galaxies from Gerin \& Phillips (2000). Arrows mark $3 \sigma$ upper limits on $L_{[\mathrm{C}]]^{3} P_{1}-{ }^{3} P_{0}}$. Right: $M_{[\mathrm{C} \mathrm{]}]} / M_{\mathrm{dust}}$ as a function of $L_{\mathrm{IR}}$. Symbols are coded as in Figure 2 and reported in the legend. Open red circles mark sources without a reliable continuum detection in the Rayleigh-Jeans tail, thus having an uncertain dust mass. In both panels, the $L_{\mathrm{IR}}$ of QSOs includes the emission of the dusty tori, at odds with the rest of the SF-dominated galaxies.

at $z \sim 1.2$, local LIRGs, and high-redshift SMGs within a fairly large scatter. We estimate a mean value of log $\left(L_{[\mathrm{C} I]^{3} P_{1}-{ }^{3} P_{0}}^{\prime} / L_{\mathrm{CO}(2-1)}^{\prime}\right)=(-0.69 \pm 0.04) \quad$ with an observed scatter of 0.23 dex for $37>3 \sigma$ detected galaxies, largely dominated by the intrinsic dispersion of 0.20 dex. The inclusion of six upper limits with a survival analysis provides consistent values (the mean from the Kaplan \& Meier 1958 estimator is $\log \left(L_{[\mathrm{C}]]^{3} P_{1}-{ }^{3} P_{0}}^{\prime} / L_{\mathrm{CO}(2-1)}^{\prime}\right)=(-0.72 \pm 0.04)$, with

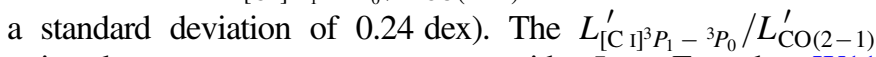
ratio does not appear to vary with $L_{\mathrm{IR}}$. For the W11 sample, we converted $\mathrm{CO}(3-2)$ to $\mathrm{CO}(2-1)$ by applying $r_{32}=L_{\mathrm{CO}(3-2)}^{\prime} / L_{\mathrm{CO}(2-1)}^{\prime}=0.62$ (Bothwell et al. 2013). Adopting ratios close to those of the original W11 paper $\left(r_{32}=1\right.$ for fully thermalized gas) would result in ratios $\sim 60 \%$ larger, bringing them closer to the values from B17. For the FTS sample, we used the observed $\mathrm{CO}(2-1)$ luminosities when available (26/32 galaxies) and converted $\mathrm{CO}(1-0)$ to $\mathrm{CO}(2-1)$, fixing $r_{21}=L_{\mathrm{CO}(2-1)}^{\prime} / L_{\mathrm{CO}(1-0)}^{\prime}=0.84$ for three more sources. These ratios are consistent with the observations in local spirals, mergers, and low-metallicity galaxies and their large scatter reported in Gerin \& Phillips (2000), once corrected for the small excitation bias between $\mathrm{CO}(2-1)$ and $\mathrm{CO}(1-0)\left(\log \left(L_{[\mathrm{C} I]^{3} P_{1}-{ }^{3} P_{0}}^{\prime} / L_{\mathrm{CO}(2-1)}^{\prime}\right)=-0.7 \pm 0.4\right.$ dex, with extreme values of $\log \left(L_{[\mathrm{C} 1]^{3} P_{1}-{ }^{3} P_{0}}^{\prime} / L_{\mathrm{CO}(2-1)}^{\prime}\right) \sim-1.4$ and 0$)$. Therefore, [C I] $\left({ }^{3} P_{1}-{ }^{3} P_{0}\right)$ and low-J CO transitions appear to be correlated on $>\mathrm{kpc}$ scales regardless of galaxy type, total $L_{\mathrm{IR}}$, and redshift, although with substantial scatter due to object-by-object variations, given the small measurement errors on fluxes.

\subsection{Neutral Atomic Carbon Masses}

Following Weiß et al. (2005), the mass of atomic carbon is derived straightforwardly from $L_{[\mathrm{C} I]^{3} P_{1}-{ }^{3} P_{0}}$ as

$$
M_{[\mathrm{C} \text { i }]}=5.706 \times 10^{-4} Q\left(T_{\mathrm{ex}}\right) \frac{1}{3} \mathrm{e}^{23.6 / T_{\mathrm{ex}}} L_{[\mathrm{C} \mathrm{I}]{ }^{3} P_{1}-{ }^{3} P_{0}}^{\prime}\left[M_{\odot}\right],
$$

where $Q\left(T_{\text {ex }}\right)=1+3 \mathrm{e}^{-23.6 \mathrm{~K} / T_{\text {ex }}}+5 \mathrm{e}^{-62.5 \mathrm{~K} / T_{\text {ex }}}$ is the partition function of [C I ] and $T_{\mathrm{ex}}$ is the excitation temperature. We

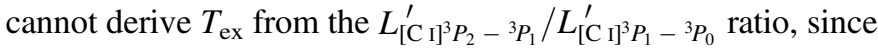
[C I] $\left({ }^{3} P_{2}-{ }^{3} P_{1}\right)$ is unavailable for our sample of MS galaxies at $z \sim 1.2$. Therefore, we assume a fixed [CI] excitation temperature $T_{\mathrm{ex}}=30 \mathrm{~K}$ for all galaxies. W11 reported a $\left\langle T_{\mathrm{ex}}\right\rangle=29.1 \pm 6.3 \mathrm{~K}$ for their overall sample of SMGs and QSOs, and we derive $\left\langle T_{\mathrm{ex}}\right\rangle=25 \pm 1 \mathrm{~K}$ for part of the FTS local sample with both [C I] $\left({ }^{3} P_{1}-{ }^{3} P_{0}\right)$ and [C I] $\left({ }^{3} P_{2}-{ }^{3} P_{1}\right)$. A typical value of $30 \mathrm{~K}$ was also previously adopted by AZ13 and B17. Assuming the dust temperature that we estimate from the far-infrared SED modeling as a first guess for the [C I] $T_{\mathrm{ex}}$, we find similar results $\left(\left\langle T_{\text {dust }}\right\rangle=31 \pm 1 \mathrm{~K}\right.$ for the MS calibration sample and $\left\langle T_{\text {dust }}\right\rangle=36 \pm 2 \mathrm{~K}$ for the SBs). Note that the total neutral carbon mass is insensitive to $T_{\mathrm{ex}}$ above $20 \mathrm{~K}$ (Weiß et al. 2005), so the exact choice of this parameter is not critical above this threshold, which is unlikely in the samples studied here. We report the total M[CI] masses for our galaxies at $z \sim 1.2$, the local FTS sample, and high-redshift SMGs/QSOs in Tables 2, 4, 5, and 6. 


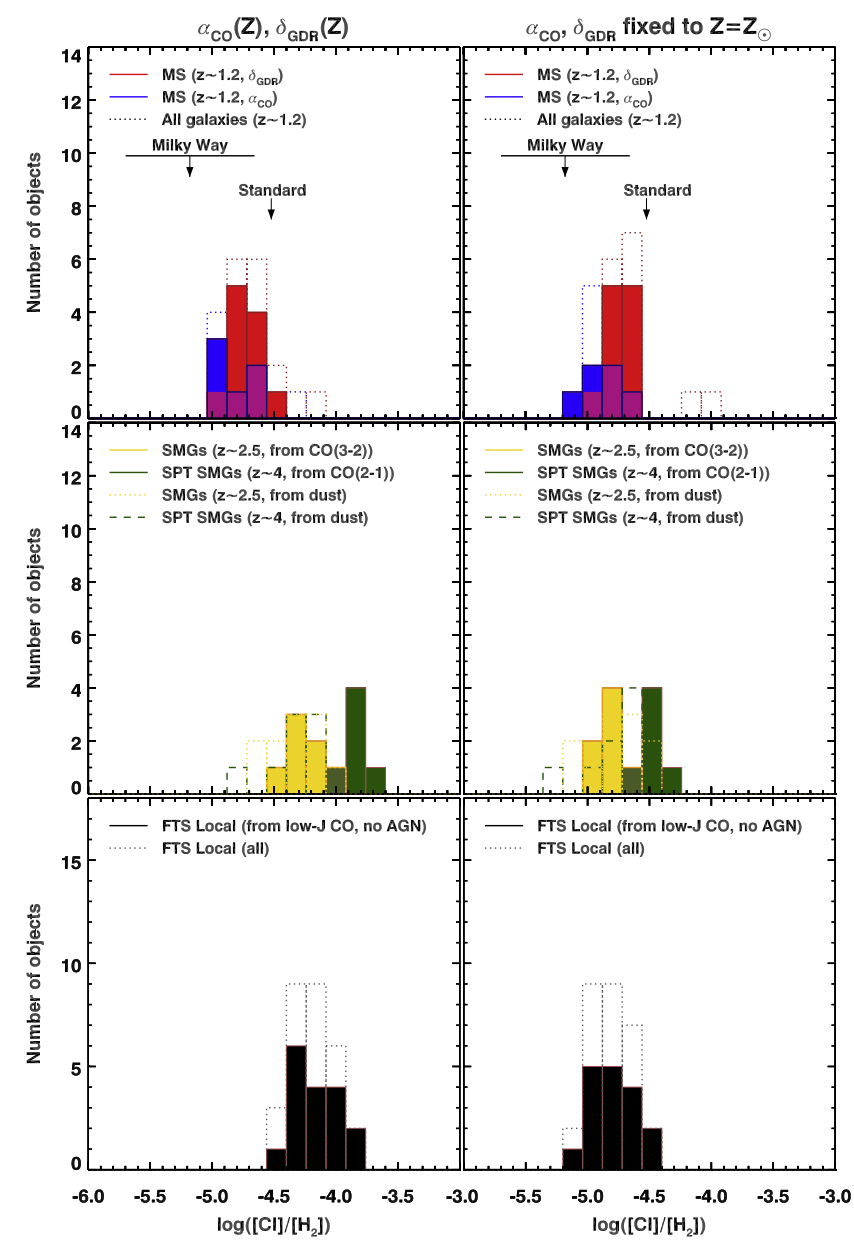

Figure 5. Atomic carbon abundances. Top: The red and blue filled histograms show the distribution of the carbon abundances $[\mathrm{C} \mathrm{I}] /\left[\mathrm{H}_{2}\right]$ for our sample of MS galaxies with $>3 \sigma$ detections of $[\mathrm{C} \mathrm{I}]\left({ }^{3} P_{1}-{ }^{3} P_{0}\right)$ derived using a dust- and $\mathrm{CO}(2-1)$-based $M_{\mathrm{H}_{2}}$ (Section 4.4). The black segment and arrows show the range of values reported in the literature for regions of the Milky Way $\left(\log \left([\mathrm{C} \mathrm{I}] /\left[\mathrm{H}_{2}\right]\right)=(-5.7,-4.7)\right.$; Frerking et al. 1989) and the commonly adopted value of $\log \left([\mathrm{C} \mathrm{I}] /\left[\mathrm{H}_{2}\right]\right)=-4.5$ (Weiß et al. 2003). The dotted lines mark the distributions, including SBs and AGNs, when possible. Center: The yellow and green filled (dashed/dotted) histograms show $[\mathrm{C} \mathrm{I}] /\left[\mathrm{H}_{2}\right]$ for SMGs from W11/AZ13 and B17 using a $\mathrm{CO}(2-1)$ (dust)-based $M_{\mathrm{H}_{2}}$. Bottom: The black filled histograms show $[\mathrm{C}] /\left[\mathrm{H}_{2}\right]$ for local LIRGs without AGN signatures using CO-based $M_{\mathrm{H}_{2}}$. The dotted histogram shows the abundance distribution for the whole FTS sample, including active galaxies. Left panels: $M_{\mathrm{H}_{2}}$ derived adopting metallicity-dependent $\alpha_{\mathrm{CO}}$ and $\delta_{\mathrm{GDR}}$ for MS galaxies and fixed $\alpha_{\mathrm{CO}}=0.8 M_{\odot} /\left(\mathrm{K} \mathrm{km} \mathrm{s}^{-1} \mathrm{pc}^{2}\right)$ and $\delta_{\mathrm{GDR}}=30$ for SBs, local FTS galaxies, and SMGs. Right panels: universal $\alpha_{\mathrm{CO}}=3.3$ $M_{\odot} /\left(\mathrm{K} \mathrm{km} \mathrm{s}^{-1} \mathrm{pc}^{2}\right)$ and $\delta_{\mathrm{GDR}}=85$, following Magdis et al. (2012b) and fixing $Z=Z_{\odot}$ for all galaxies.

\subsection{Gas Masses from Dust and CO}

We estimated the total gas masses (including a $1.36 \times$ contribution from helium) using both dust masses from SED modeling and $\mathrm{CO}(1-0)$ or $\mathrm{CO}(2-1)$ as gas tracers, when available. In both cases, we adopted a metallicity-dependent conversion factor as described in Magdis et al. (2012b). ${ }^{15}$ Note that this approach includes the atomic hydrogen $\mathrm{H}$ I in the gas mass estimate, a significant contributor to the total gas mass only at low redshift. We derived metallicities converting stellar

\footnotetext{
15 Magdis et al. (2012b) reported an intercept of 12.8 in their parameterization of $\alpha_{\mathrm{CO}}(12+\log (\mathrm{O} / \mathrm{H})$ ) (Equation (8)), not matching their Figure 5 (right). The correct value adopted here is 12.6. The parameterization of $\delta_{\mathrm{GDR}}(12+\log (\mathrm{O} / \mathrm{H}))$ is identical to that of Magdis et al.
}

masses and SFR with the fundamental metallicity relation (Mannucci et al. 2010). The derived metallicities are generally consistent with the solar value $\left(12+\log (\mathrm{O} / \mathrm{H})_{\odot}=8.69\right.$; Asplund et al. 2009). We estimate an average gas-to-dust conversion factor of $\left\langle\delta_{\mathrm{GDR}}\right\rangle \sim 87$ and $\left\langle\alpha_{\mathrm{CO}}\right\rangle \sim 3 M_{\odot} /$ $\left(\mathrm{K} \mathrm{km} \mathrm{s}^{-1} \mathrm{pc}^{2}\right)$. We assumed an $L_{\mathrm{CO}(2-1)}^{\prime} / L_{\mathrm{CO}(1-0)}^{\prime}$ ratio of $r_{21}=0.84$ to convert $\mathrm{CO}(2-1)$ into total gas masses when necessary (Magdis et al. 2012b; Bothwell et al. 2013). We further derived total gas masses for our SBs at $z \sim 1.2$, LIRGs and high-redshift SMGs fixing the conversion factors to $\alpha_{\mathrm{CO}}=0.8 M_{\odot} /\left(\mathrm{K} \mathrm{km} \mathrm{s}^{-1} \mathrm{pc}^{2}\right)$, and $\delta_{\mathrm{GDR}}=30$. The final error budget includes the uncertainties on the observed $\mathrm{CO}$ fluxes and dust mass from SED modeling (Section 2.2). We further include a 0.2 dex statistical error on $\alpha_{\mathrm{CO}}$ and $\delta_{\mathrm{GDR}}$, mimicking the uncertainty on the metallicity-dependent parameterization in Magdis et al. (2012b). Possible larger systematic uncertainties affecting the gas masses are not listed in the error budget (e.g., see Kamenetzky et al. 2017 for a study of the local LIRGs).

Figure 4 shows that the $M_{[\mathrm{C}]} / M_{\text {dust }}$ ratios of SMGs appear similar to the values for our MS and SB galaxies at $z \sim 1.2$, albeit with substantial scatter. We estimate a mean ratio of $\log \left(M_{[\mathrm{C} \mathrm{I}} / M_{\text {dust }}\right)=(-2.20 \pm 0.03)$, with an observed scatter of $\sigma=0.19$ dex dominated by an intrinsic dispersion of 0.15 dex for 33 galaxies with a $>3 \sigma$ detection of [C I] $\left({ }^{3} P_{1}-{ }^{3} P_{0}\right)$. The inclusion of 11 upper limits with a survival analysis provides a consistent result $\left(\log \left(M_{\left[\mathrm{C}_{\mathrm{I}}\right]} / M_{\text {dust }}\right)=\right.$ $(-2.26 \pm 0.04), \sigma=0.23 \mathrm{dex})$. Note that we excluded active galaxies, QSOs, and galaxies without a detection of the dust continuum from this calculation. Moreover, the SMGs from $\mathrm{B} 17$ at $z \sim 4$ appear to have fainter $\mathrm{CO}(2-1)$ emission than the sample from W11 at $z \sim 2.5$ at fixed dust mass, assuming the SLED ratios from Bothwell et al. (2013; Section 4.2 of this paper.). This is likely the result of a combination of different factors, including the gas excitation properties of individual SMGs, a redshift effect due to the evolution of the strength of the radiation field $\langle U(z)\rangle \propto L_{\mathrm{IR}} / M_{\mathrm{dust}}$, the different selection techniques, and the heterogeneity of the SMG population (Carilli \& Walter 2013).

\subsection{The Abundance of Neutral Atomic Carbon in Galaxies}

While not requiring a standard $\alpha$ or $X$ factor as optically thick ${ }^{12} \mathrm{CO}$ transitions, [C I] line luminosities can be converted into total gas masses only with prior knowledge of the abundance of carbon in the neutral atomic phase $[\mathrm{CI}] /\left[\mathrm{H}_{2}\right]$. Such a conversion is necessary for any species other than $\mathrm{H}_{2}$, the dominant form of molecular gas. We derived the atomic carbon abundances as $[\mathrm{C} \mathrm{I}] /\left[\mathrm{H}_{2}\right]=M_{[\mathrm{C}]} /\left(6 M_{\mathrm{H}_{2}}\right)$ using the $M_{\mathrm{H}_{2}}$ estimates from dust and CO. Notice that $M_{\mathrm{H}_{2}}$ does not include the helium contribution. Previous works adopted this or alternative approaches, providing atomic carbon abundance estimates in a variety of environments at different redshifts (e.g., Stutzki et al. 1997; Ikeda et al. 2002; Weiß et al. 2003, 2005; Israel et al. 2015, W11, AZ13 and B17 among the others). Here we redetermined the abundances based on a homogeneous set of assumptions to directly compare data sets in a consistent way. The discrepancies among our estimates and the ones in the original papers arise mainly from the choice of galaxies representative of the various populations (e.g., we exclude QSOs from the calculations) and different assumptions (gas conversion factors, $\mathrm{CO}$ excitation ladder, inclusion or not of upper limits, dust- or CO-based gas masses, etc). We show 
the distribution of the estimated abundances for the MS sample at $z \sim 1.2$ in Figure 5. The mean values are $\log \left([\mathrm{C} \mathrm{I}] /\left[\mathrm{H}_{2}\right]\right)=$ $(-4.7 \pm 0.1)$ and $(-4.8 \pm 0.2)$ adopting $M_{\mathrm{H}_{2}}$ (dust) and $M_{\mathrm{H}_{2}}(\mathrm{CO})$, respectively. The uncertainties represent the standard deviation of the observed distributions for $12 \mathrm{MS}$ galaxies with detected continuum emission in the Rayleigh-Jeans tail and [C I] $\left({ }^{3} P_{1}-{ }^{3} P_{0}\right)$ coverage (11 detections and one upper limit) when using $M_{\mathrm{H}_{2}}$ (dust) and for eight objects with [C I] $\left({ }^{3} P_{1}-{ }^{3} P_{0}\right)$ coverage (six detections and two upper limits) and $\mathrm{CO}(2-1)$ detections in the case of $M_{\mathrm{H}_{2}}(\mathrm{CO})$. We excluded one MS source with continuum detection due to unsuccessful far-infrared photometric deblending. We included the upper limits on $[\mathrm{CI}]\left({ }^{3} P_{1}-{ }^{3} P_{0}\right)$ using a survival analysis, but their effect is negligible. The $[\mathrm{C} \mathrm{I}] /\left[\mathrm{H}_{2}\right]$ in MS galaxies at $z \sim 1.2$ is consistent with the observed values in highly obscured clouds of the Milky Way $\left(\log \left([\mathrm{C} \mathrm{I}] /\left[\mathrm{H}_{2}\right]\right) \sim-5.7,-4.7\right.$, depending on the dust attenuation; Frerking et al. 1989, Figure 5 of this paper), and it is lower than the typically assumed abundance of $\log \left([\mathrm{C} \mathrm{I}] /\left[\mathrm{H}_{2}\right]\right)=-4.5$ (Weiß et al. 2003). For reference, we also show the distributions including SBs and AGN-dominated objects. We remark that the abundances presented in this work are global, galaxy-integrated estimates, while local measurements often focus on individual clouds. A direct comparison should be drawn with caution, as it would be natural to find lower abundances on global scales if [C I] and $\mathrm{H}_{2}$ are not fully cospatial. We similarly rederived the atomic carbon abundances for the literature sample using both $M_{\mathrm{H}_{2}}(\mathrm{CO})$ and $M_{\mathrm{H}_{2}}$ (dust) when available. For the local sample of 17 sources without AGN signatures and $\mathrm{CO}(1-0)$ detections, we find $\log \left([\mathrm{C} \mathrm{I}] /\left[\mathrm{H}_{2}\right]\right)=(-4.2 \pm 0.2)$; for the SPT SMGs at $z \sim 4$ from $\mathrm{B} 17, \log \left([\mathrm{C} \mathrm{I}] /\left[\mathrm{H}_{2}\right]\right)=(-3.9 \pm 0.1)$ and $(-4.3 \pm 0.2)$ using $\mathrm{CO}(2-1)$ and dust, respectively; and for SMGs at $z>2.5$ from W11 and AZ13, $\log \left([\mathrm{C} \mathrm{I}] /\left[\mathrm{H}_{2}\right]\right)=(-4.2 \pm 0.1)$ and $(-4.3 \pm 0.2)$ using $\mathrm{CO}(2-1)$ and dust, respectively. The uncertainties represent the dispersion of the distributions in Figure 5 and include upper limits with a survival analysis. Note that $[\mathrm{C} \mathrm{I}] /\left[\mathrm{H}_{2}\right]=M_{[\mathrm{C}]} /\left(6 M_{\mathrm{H}_{2}}\right)$ faithfully represents the abundance of atomic carbon relative to the molecular hydrogen $\mathrm{H}_{2}$, a good approximation for the total gas mass $M_{\text {gas }}$ at high redshift. However, both $M_{\mathrm{H}_{2}}$ (dust) and $M_{\mathrm{H}_{2}}(\mathrm{CO})$ formally include $\mathrm{HI}$, which might be the dominant phase in local systems. Removing $\mathrm{HI}$ and considering the molecular gas phase only would further increase the $[\mathrm{CI}] /\left[\mathrm{H}_{2}\right]$ values reported above for the local galaxies.

\section{The Calibration of $[\mathrm{C} \mathrm{I}]$ as a Gas Tracer in Galaxies}

\subsection{The Limitations of a Fully Empirical Approach}

So far, we have proven that it is feasible to detect [C I] $\left({ }^{3} P_{1}-{ }^{3} P_{0}\right)$ lines not only in distant, extreme, and often lensed systems, such as SMGs and QSOs, but also in normal MS galaxies at moderately high redshifts. We showed the existence of an $L_{\mathrm{IR}}-L_{[\mathrm{C} \mathrm{I}]^{3} P_{1}-{ }^{3} P_{0}}^{\prime}$ correlation similar to the standard

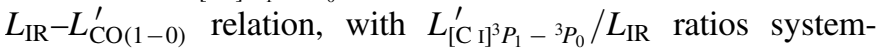
atically decreasing with increasing $L_{\mathrm{IR}}$ and distance from the main sequence. The strong correlation between $L_{[\mathrm{C} I]^{3} P_{1}-{ }^{3} P_{0}}^{\prime}$ and $L_{\mathrm{IR}}$ makes the latter a useful tool to predict [C I] emission in distant galaxies. Moreover, the roughly constant $L_{[\mathrm{C}]^{3} P_{1}-{ }^{3} P_{0}}^{\prime} / L_{\mathrm{CO}(2-1)}^{\prime}$ and $M_{[\mathrm{C} \mathrm{I}]} / M_{\text {dust }}$ ratios on $\mathrm{kpc}$ scales, regardless of total $L_{\mathrm{IR}}$, galaxy type, and redshift, reinforce the connection between [C I], $\mathrm{CO}$, and dust, supporting the use of [C I] as a molecular gas tracer.

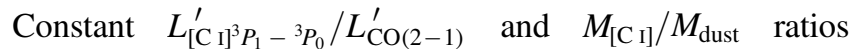
directly translate into systematically lower neutral atomic carbon abundances in MS galaxies than in SBs/SMGs, owing to the canonical empirical $\alpha_{\mathrm{CO}}$ and gas-to-dust $\delta_{\mathrm{GDR}}$ conversion factors $\left(\alpha_{\mathrm{CO}} \sim 4-0.8 M_{\odot} /\left(\mathrm{K} \mathrm{km} \mathrm{s}^{-1} \mathrm{pc}^{2}\right)\right.$ and $\delta_{\mathrm{GDR}} \sim 85-30$ for $\mathrm{MS}$ and $\mathrm{SB}$ galaxies, respectively). Assuming identical conversion factors, the abundances are similar in MS galaxies and SBs/SMGs (we show the case of constant $\alpha_{\mathrm{CO}}, \delta_{\mathrm{GDR}}\left(Z=Z_{\odot}\right)$ in Figure 5). In other words, the well-known uncertainties of the standard $\mathrm{CO}$ and dust tracers affect the empirical calibration of [C I]. This practically limits the use of this potentially superior tracer of gas in galaxies in the absence of a calibration fully independent of the current assumptions. Moreover, these results suggest that the use of a universal abundance at low and high redshift, and regardless of the galaxy population, can strongly bias the gas masses derived from $[\mathrm{C} \mathrm{I}]\left(M_{\mathrm{H}_{2}}([\mathrm{CI}])\right.$ scales as $\left.\left([\mathrm{CI}] /\left[\mathrm{H}_{2}\right]\right)^{-1}\right)$, as in the case of the widespread $[\mathrm{C} \mathrm{I}] /\left[\mathrm{H}_{2}\right]=3 \times 10^{-5}$ value adopted in the literature, following an estimate by Weiß et al. (2003) in a high-redshift QSO and the average abundance reported by Papadopoulos \& Greve (2004). The ascertained redshift evolution of metallicity in galaxies and the complex history of carbon production (e.g., Chiappini et al. 2003) argue against the use of universal abundance values, even if an early and quick enrichment might mitigate this issue in the cosmic ages explored so far.

\subsection{The Small Fraction of Atomic Carbon in Galaxies}

A precise calibration of [C I] as a total gas tracer could be intrinsically difficult, since it requires the tracking of the small fraction of carbon produced in galaxies in the atomic gas phase. Neglecting the carbon locked into stars, the gas mass fraction of the neutral atomic carbon phase $f_{[\mathrm{CI}]}=M_{[\mathrm{CI}]} / M_{\mathrm{C}}$ can be derived from the definition of the mass fraction of metals,

$$
Z=\frac{M_{\text {metals }}}{M_{\mathrm{H}_{2}}}=\frac{M_{\text {metals }}}{M_{\mathrm{C}}} \frac{M_{\mathrm{C}}}{M_{[\mathrm{C} \mathrm{I}]}} \frac{M_{[\mathrm{C} \mathrm{I}]}}{M_{\mathrm{H}_{2}}},
$$

where $M_{\mathrm{C}}$ is the total mass of carbon, $M_{[\mathrm{C} \text { I] }}$ is the mass in the neutral atomic phase, $M_{\text {metals }}$ is the total mass of metals, and $M_{\mathrm{H}_{2}}$ is the hydrogen gas mass, excluding helium. From Equation (3), we derive $f_{[\mathrm{C} \mathrm{I}]} \propto \frac{M_{[\mathrm{CI}]}}{M_{\mathrm{dust}} \delta_{\mathrm{GDR}} / \mathrm{He}}$ or $\propto \frac{M_{[\mathrm{CI}]}}{L_{\mathrm{CO}}^{\prime} \alpha_{\mathrm{CO}} / \mathrm{He}}$. Assuming a solar metallicity and composition $\left(Z_{\odot}=0.0134\right.$ and $\frac{M_{\mathrm{C}}}{M_{\text {metals }}}=0.1779$; Asplund et al. 2009), the mass fraction of carbon in the atomic gas phase in the MS galaxies is $4.6_{-1.0 \%}^{+1.3 \%}$ and $3.4_{-1.0 \%}^{+1.4 \%}$ using $M_{\text {dust }}$ and $L_{\mathrm{CO}(2-1)}^{\prime}$, respectively (Figure 6). These estimates and their uncertainties include upper limits through survival analysis, and they represent the mean and standard deviation of the logarithmic distributions in Figure 6. Note that these values depend on the choice of metallicity and carbon fraction adopted here. Using the metallicity from the fundamental metallicity relation does not impact this result (Section 4.4). We derive similar $f_{\left[\mathrm{C}_{\mathrm{I}}\right]}=6.0_{-2.1}^{+3.3} \%$ for LIRGs; $4.5_{-1.7}^{+2.8} \%$ and $5.3_{-1.4}^{+1.9} \%$ for SMGs at $z \sim 2.5$ from W11/AZ13 using dust and CO, respectively; and $4.3_{-1.6}^{+2.6} \%$ and $12.7_{-2.6}^{+3.2} \%$ for SPT SMGs from B17 adopting the dust- and CO-based calibration and including upper limits. We used dust and $\mathrm{CO}$ to derive $M_{\mathrm{gas}}$ and assuming a metallicity of $Z \sim 2.8 Z_{\odot}$ corresponding to $\alpha_{\mathrm{CO}}=0.8 M_{\odot} /\left(\mathrm{K} \mathrm{km} \mathrm{s}^{-1} \mathrm{pc}^{2}\right)$ and $\delta_{\mathrm{GDR}}=30$. 


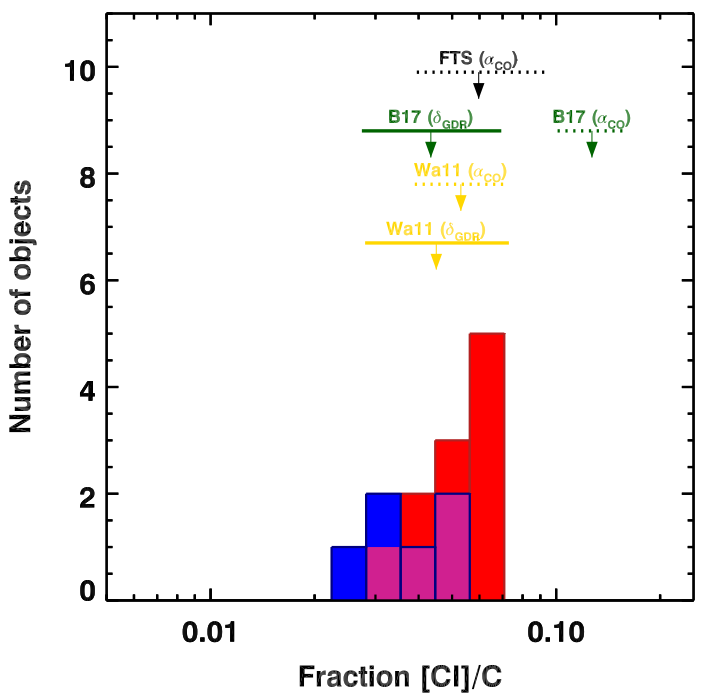

Figure 6. Atomic carbon fractions. The red and blue filled histograms show the distribution of the neutral atomic carbon mass fractions $f_{[\mathrm{C}]}=M_{[\mathrm{C} \mathrm{I}]} / M_{\mathrm{C}}$ for our sample of MS galaxies with $>3 \sigma$ detections of [C I] $\left({ }^{3} P_{1}-{ }^{3} P_{0}\right)$ derived using a dust- and $\mathrm{CO}(2-1)$-based $M_{\mathrm{H}_{2}}$, adopting $\delta_{\mathrm{GDR}}(Z)=\delta_{\mathrm{GDR}}\left(Z_{\odot}\right)$ and $\alpha_{\mathrm{CO}}(Z)=\alpha_{\mathrm{CO}}\left(Z_{\odot}\right)$. The colored segments and arrows show the mean $f_{[\mathrm{C} \text { I] }}$ values and the standard deviation of the logarithmic distributions of SMGs and local LIRGs, as coded in the labels (B17: SPT SMGs at $z \sim 4$; Wa11: SMGs at $z \sim 2.5$; FTS: local LIRGs, no AGNs).

Supersolar metallicities are necessary to obtain these commonly adopted values when using the parameterization by Magdis et al. (2012b; their Equation (8), with the corrected intercept discussed above and in Section 4.2). However, while this likely has strong physical roots for the optically thin dust emission, $\alpha_{\mathrm{CO}}$ values of $\sim 0.8 M_{\odot} /\left(\mathrm{K} \mathrm{km} \mathrm{s}^{-1} \mathrm{pc}^{2}\right)$ could also be found at $Z \sim Z_{\odot}$, the $\mathrm{CO}$ emission being optically thick and thus critically dependent on other parameters (i.e., the FWHM of the line).

The atomic carbon fractions $f_{\left[\mathrm{C}_{\mathrm{I}}\right]}$ in Figure 6 reflect the similar $L_{[\mathrm{C}]^{3} P_{1}-{ }^{3} P_{0}}^{\prime} / L_{\mathrm{CO}(2-1)}^{\prime}$ and $M_{[\mathrm{C} \mathrm{I}]} / M_{\text {dust }}$ ratios for MS and SBs/SMGs in Figure 4, analogous to the [C I] abundances shown in Figure 5. From a theoretical perspective, even simple plane-parallel PDR models could partially explain the observed constant [CI] $\left({ }^{3} P_{1}-{ }^{3} P_{0}\right) / \mathrm{CO}(2-1)$ ratio by the relative insensitivity of the $[\mathrm{CI}]$ emission to the strength of $\mathrm{UV}$ radiation. In fact, while a stronger radiation field pushes the C-to-CO transition deeper into the gas slab, the size of the [C I]and $\mathrm{CO}(2-1)$-emitting regions stays relatively constant (e.g., Kaufman et al. 1999 and many others). Our $f_{\left[\mathrm{C}_{\mathrm{I}}\right]}$ estimates suggest that [C I] represents a very minor fraction of the overall mass of carbon in galaxies, as the majority is in $\mathrm{CO}$ molecules $(N([\mathrm{C} \mathrm{I}]) / N(\mathrm{CO})=0.1-0.2$; Ikeda et al. 2002) and depleted on dust ( $\sim 27 \%$ of the overall carbon abundance; e.g., van Dishoeck \& Black 1988), neglecting the quantity locked in stars. The small mass fraction of atomic carbon and the associated low column densities explain the small optical depth of the $[\mathrm{C} \mathrm{I}]\left({ }^{3} P_{1}-{ }^{3} P_{0}\right)$ line, a major advantage in the use of this species to trace the gas content of galaxies (Papadopoulos et al. 2004). However, an accurate assessment of such minimal [CI] fractions and the detection of relative variations in different galaxy populations-if present-are complicated by both observational and theoretical uncertainties (e.g., the history of chemical enrichment in galaxies, affecting any tracers of the molecular gas mass other than $\mathrm{H}_{2}$ ).

\subsection{Possible Hidden Systematic Variations of $[\mathrm{C} \mathrm{I}] /\left[\mathrm{H}_{2}\right]$}

We showed that the MS and SB/SMGs have similar $L_{[\mathrm{C}]{ }^{3} P_{1}-{ }^{3} P_{0}}^{\prime} / L_{\mathrm{CO}(2-1)}^{\prime}$ and $M_{[\mathrm{C} \mathrm{I}]} / M_{\text {dust }}$ ratios (Figure 4$)$, resulting in different $[\mathrm{C} \mathrm{I}] /\left[\mathrm{H}_{2}\right]$ owing to standard assumptions on the dust/CO-to-gas conversion factors. However, reversing the argument, intrinsic large variations of $[\mathrm{C} \mathrm{I}] /\left[\mathrm{H}_{2}\right]$ might not directly translate into large differences in the observed ratios, due to the countereffect of higher $\delta_{\mathrm{GDR}}$ and $\alpha_{\mathrm{CO}}$ for MS galaxies than for SBs. Analogously, intrinsic systematic differences of $[\mathrm{CI}] /\left[\mathrm{H}_{2}\right]$ between the $\mathrm{SB}$ and $\mathrm{MS}$ would blur large variations of SFE into similar $L_{\mathrm{IR}} / L_{[\mathrm{C} \mathrm{I}]^{3} P_{1}-{ }^{3} P_{0}}$ ratios, equivalent to what is observed for CO (Daddi et al. 2010b). The opposite dependence of $[\mathrm{CI}] /\left[\mathrm{H}_{2}\right]$ and $\delta_{\mathrm{GDR}}$ on metallicity could explain the small variations of $M_{\left[\mathrm{C}_{\mathrm{I}}\right]} / M_{\text {dust }}$ observed in our data compilation: metal-rich $\mathrm{SB}$ galaxies tend to have larger $[\mathrm{CI}] /\left[\mathrm{H}_{2}\right]$ abundances than MS objects, compensating for lower gas-to-dust ratios, $\delta_{\mathrm{GDR}}$. On the other hand, parameters other than metallicity should play a major role in the comparison of optically thick (CO) and thin ([C I], dust) tracers (e.g., turbulent velocities and compression resulting in broad CO lines; Bournaud et al. 2015). On top of these effects, enhanced cosmic-ray rates in sources with large SFEs (e.g., Papadopoulos et al. 2004; Bisbas et al. 2015, 2017) could increase $[\mathrm{C} \mathrm{I}] /\left[\mathrm{H}_{2}\right]$, further reducing strong variations of the

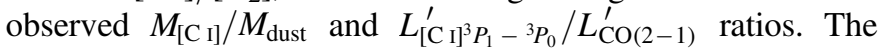
degeneracies listed here would be broken by an estimate of the gas mass independent of the assumptions we have to make for the currently available data.

\section{Conclusions}

We presented the first results of a survey targeting the $[\mathrm{C} \mathrm{I}]$ $\left({ }^{3} P_{1}-{ }^{3} P_{0}\right)$ transition in main-sequence galaxies at $z \sim 1.2$, expanding the samples of starbursts and SMGs currently present in the literature toward a population of normal galaxies.

We showed the existence of a sublinear correlation between

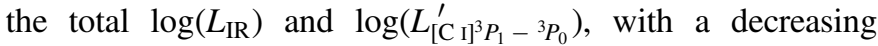
$L_{\left[\mathrm{C} I{ }^{3} P_{1}-{ }^{3} P_{0}\right.}^{\prime} / L_{\mathrm{IR}}$ ratio with increasing $L_{\mathrm{IR}}$. The slope of this relation and the $\sim 4 \sigma$ significant displacement of the [C I] $\left({ }^{3} P_{1}-{ }^{3} P_{0}\right)$-detected MS galaxies from the sequence traced by SBs/SMGs are similar to what is observed for CO $(1-0)$. The difference will be easily tested by populating the distance from

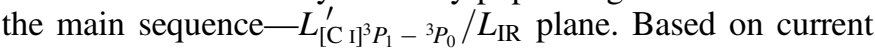
evidence, starbursts may have lower values of this ratio than main-sequence galaxies. These observations strengthen the [C I]-CO relation on global galaxy scales and suggest that

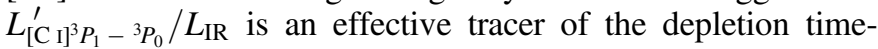
scales in galaxies, decreasing with increasing $L_{\mathrm{IR}}$ and shorter in SBs/SMGs than in MS galaxies.

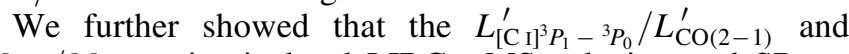
$M_{\text {[C I] }} / M_{\text {dust }}$ ratios in local LIRGs, MS galaxies, and SBs at $z \sim 1.2$ and lensed SMGs at $z>2.5-4$ are similar, with an intrinsic scatter of $\sim 0.2 \mathrm{dex}$. These ratios are proportional to a mass fraction of carbon in the neutral atomic phase of $f_{\left[\mathrm{C}_{\mathrm{I}}\right]}=M_{[\mathrm{C} \mathrm{I}]} / M_{\mathrm{C}} \sim 3 \%-13 \%$, roughly independent of the galaxy type and redshift, although affected by substantial scatter. The relative insensitivity of [CI] emission to the strength of the radiation field could partially explain the constant $[\mathrm{CI}]\left({ }^{3} P_{1}-{ }^{3} P_{0}\right) / \mathrm{CO}(2-1)$ ratios, as the size of the $[\mathrm{CI}]-$ and $\mathrm{CO}$-emitting regions stays relatively constant in different environments. 
We then estimated the atomic carbon abundances-necessary to derive the total gas mass from [C I] observations-by comparing [C I] $\left({ }^{3} P_{1}-{ }^{3} P_{0}\right)$, dust, and $\mathrm{CO}$ low- $J$ emissions. Adopting standard $\alpha_{\mathrm{CO}}$ and gas-to-dust $\delta_{\mathrm{GDR}}$ conversion factors, we find $[\mathrm{C} \mathrm{I}] /\left[\mathrm{H}_{2}\right] \sim 1.6-1.9 \times 10^{-5}$ for MS galaxies at $z \sim 1.2$. These values are $\sim 3-8 \times$ lower than the abundance in high-redshift SMGs and $\sim 4 \times$ lower than in local LIRGs. At this stage, this difference is mainly a consequence of the choice of $\alpha_{\mathrm{CO}}$ and $\delta_{\mathrm{GDR}}$, and it currently exacerbates possible minimal differences in the observables, if present. However, systematically higher $[\mathrm{C} \mathrm{I}] /\left[\mathrm{H}_{2}\right]$ in SB than in MS galaxies, possibly driven by larger metallicities and/or cosmic-ray rates, might result in similar observables, a degeneracy that cannot be broken with the available data and tools. All things considered, our findings caution against the use of a universal atomic carbon abundance regardless of the galaxy type and redshift.

We acknowledge the constructive comments from the anonymous referee that significantly improved the content and presentation of the results. We thank Mark Sargent for providing the initial catalogs and discussions during the early stages of this work. FV and GEM acknowledge the Villum Fonden research grant 13160 "Gas to stars, stars to dust: tracing star formation across cosmic time" and the Cosmic Dawn Center of Excellence funded by the Danish National Research Foundation. G.E.M. acknowledges support from the European Research Council (ERC) Consolidator Grant funding scheme (project ConTExt, grant number 648179). E.D. acknowledges funding by the Chinese Academy of Sciences Presidents International Fellowship Initiative, Grant No. 2018VMA0014. Y.G. acknowledges the partial support of the National Key R\&D Program of China grant No. 2017YFA0402704 and NSFC grant No. 11420101002. This paper makes use of the following ALMA data: ADS/JAO.ALMA\#2016.1.01040.S,
\#2015.1.00260.S, \#2016.1.00171.S. ALMA is a partnership of ESO (representing its member states), NSF (USA), and NINS (Japan), together with the NRC (Canada), MOST and ASIAA (Taiwan), and KASI (Republic of Korea), in cooperation with the Republic of Chile. The Joint ALMA Observatory is operated by the ESO, AUI/NRAO, and NAOJ. In this work, we made use of the COSMOS master spectroscopic catalog, available within the collaboration and kept updated by Mara Salvato.

\section{Appendix \\ Galaxy Spectral Energy Distributions and Spectra}

We show in Figure 7 the 1D spectra of our sample of 21 galaxies at $z \sim 1.2$ observed with ALMA Band 6. The black line shows the spectrum; the yellow area marks the channels used to measure the line fluxes, matching brighter $\mathrm{CO}$ lines, if present (Section 2.3, Table 1); the solid red line is the bestfitting Gaussian obtained by fixing the redshift and width of the [C I] $\left({ }^{3} P_{1}-{ }^{3} P_{0}\right)$ line to match the brighter $\mathrm{CO}$ emissions and the continuum level to the estimate over the full $7.5 \mathrm{GHz}$ band; and the dashed dark red line shows the best [C I] $\left({ }^{3} P_{1}-{ }^{3} P_{0}\right)$ line model with free parameters for the line and a local continuum emission estimate within $\pm 2 \mathrm{GHz}$ of the line center. When a line is not detected, we show in red the location of the expected [C I] $\left({ }^{3} P_{1}-{ }^{3} P_{0}\right)$ emission based on detected $\mathrm{CO}$ lines. The thick black tick shows the expected position of the line based on the optical/near-infrared spectroscopic redshift. Figure 7 further shows the near-infrared-to-radio photometry for our sample. The filled red circles mark the data points we considered for our modeling (black line). The open red circles were not considered in the fit (Magdis et al. 2012b). Arrows mark $3 \sigma$ upper limits. The solid blue line shows the best-fit template for the dusty torus component. 

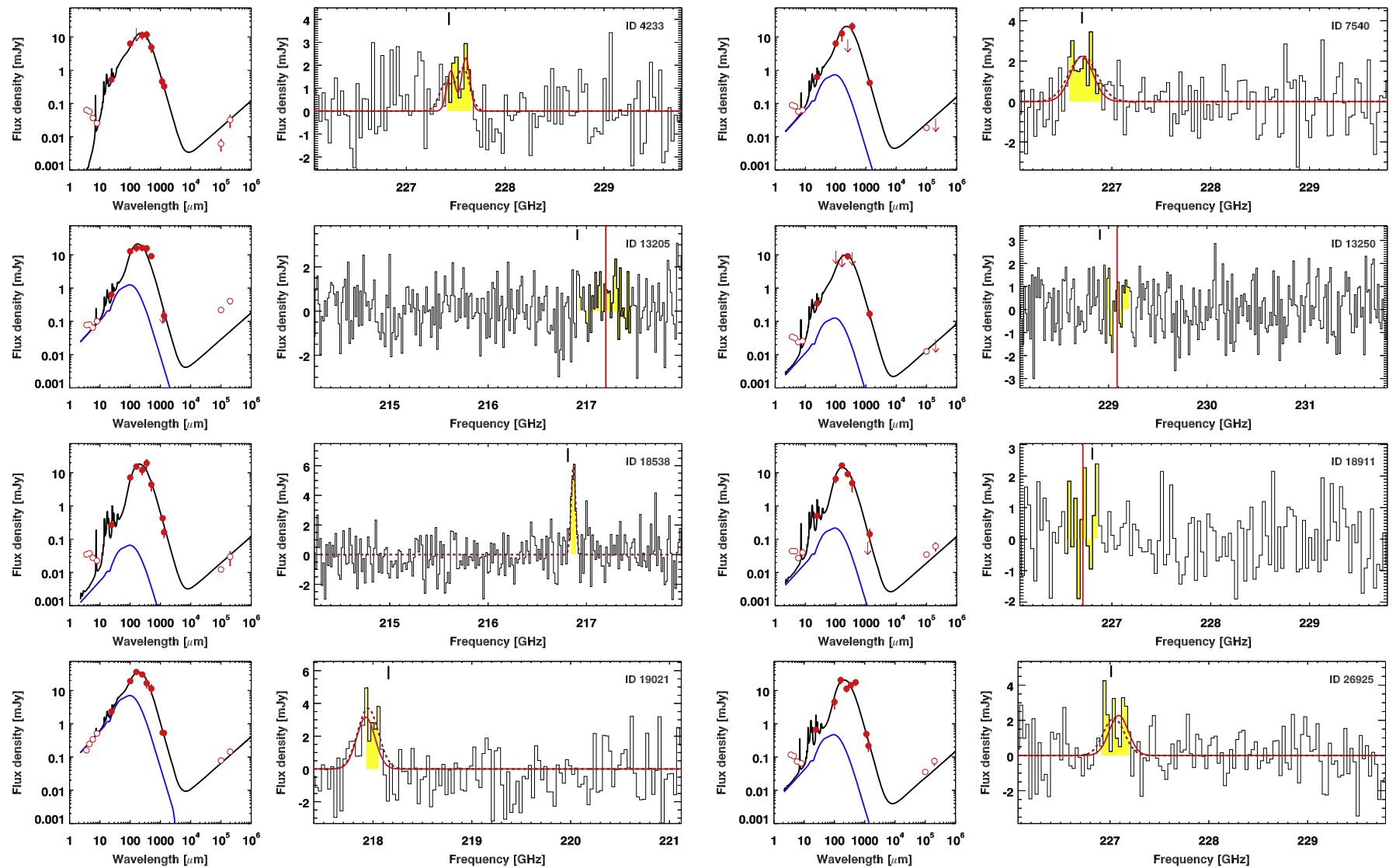

Figure 7. Far-infrared SEDs and [C I] $\left({ }^{3} P_{1}-{ }^{3} P_{0}\right)$ spectra of our sample of 21 galaxies at $z \sim 1.2$ followed up with ALMA. 

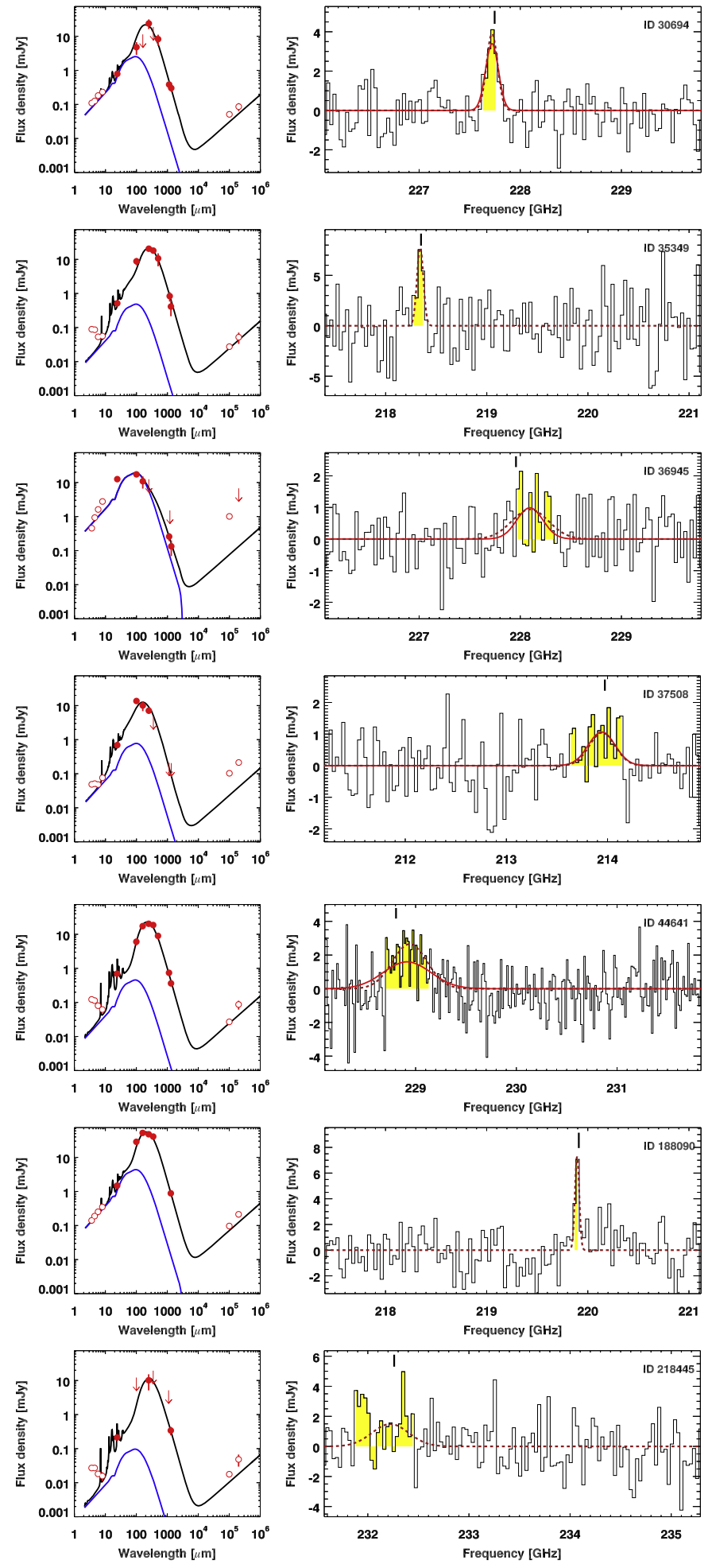

\section{ORCID iDs}

Francesco Valentino (ㄱ) https://orcid.org/0000-00016477-4011

Georgios E. Magdis (다 https://orcid.org/0000-0002-4872-2294

Emanuele Daddi (i) https://orcid.org/0000-0002-3331-9590

Daizhong Liu (1) https://orcid.org/0000-0001-9773-7479

Manuel Aravena (1) https://orcid.org/0000-0002-6290-3198

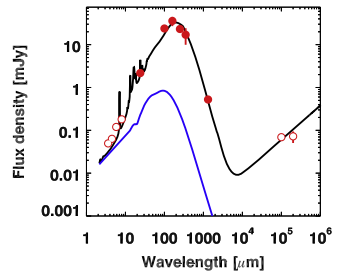

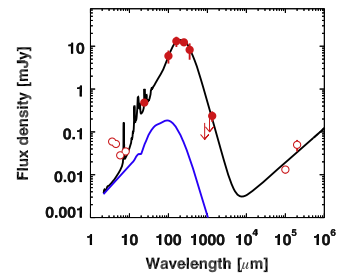
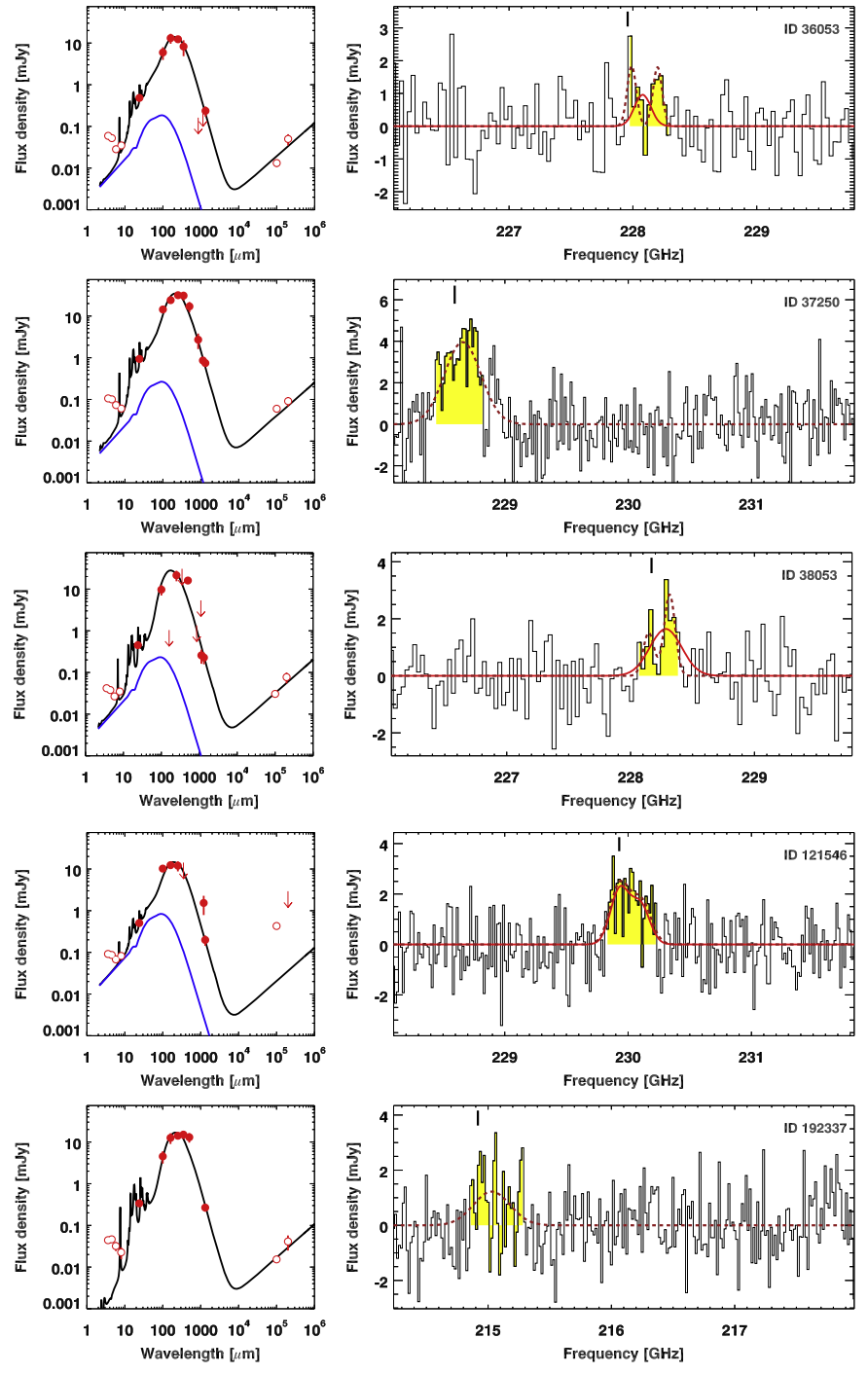

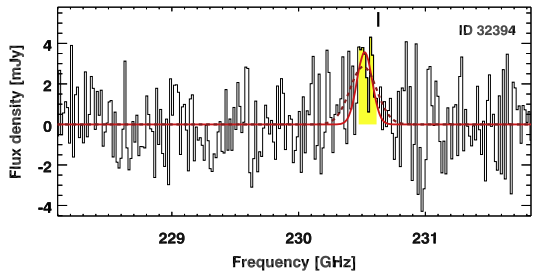

Figure 7. (Continued.)
Frédéric Bournaud @il https://orcid.org/0000-0002-5743-0250 Anna Cibinel 주 https://orcid.org/0000-0003-4578-514X Diane Cormier (ㄷ) https://orcid.org/0000-0002-1046-2685 Mark E. Dickinson (i) https://orcid.org/0000-0001-5414-5131 Yu Gao (i) https://orcid.org/0000-0003-0007-2197

Shuowen Jin (1) https://orcid.org/0000-0002-8412-7951

Stéphanie Juneau (1) https://orcid.org/0000-0002-0000-2394 Jeyhan Kartaltepe (i) https://orcid.org/0000-0001-9187-3605 
Min-Young Lee (ib https://orcid.org/0000-0002-9888-0784 Suzanne C. Madden (1) https://orcid.org/0000-00033229-2899

Annagrazia Puglisi (i] https://orcid.org/0000-0001-9369-1805

David Sanders (1) https://orcid.org/0000-0002-1233-9998

John Silverman (10) https://orcid.org/0000-0002-0000-6977

\section{References}

Alaghband-Zadeh, S., Chapman, S. C., Swinbank, A. M., et al. 2012, MNRAS, 424, 2232

Alaghband-Zadeh, S., Chapman, S. C., Swinbank, A. M., et al. 2013, MNRAS, 435, 1493

Andreani, P., Retana-Montenegro, E., Zhang, Z.-Y., et al. 2018, A\&A, 615, A142

Aravena, M., Spilker, J. S., Bethermin, M., et al. 2016, MNRAS, 457, 4406 Aretxaga, I., Wilson, G. W., Aguilar, E., et al. 2011, MNRAS, 415, 3831 Armus, L., Mazzarella, J. M., Evans, A. S., et al. 2009, PASP, 121, 559 Arnouts, S., Walcher, C. J., Le Fèvre, O., et al. 2007, A\&A, 476, 137 Asplund, M., Grevesse, N., Sauval, A. J., \& Scott, P. 2009, ARA\&A, 47, 481 Banerji, M., Jones, G. C., Wagg, J., et al. 2018, MNRAS, 479, 1154 Bertoldi, F., Carilli, C., Aravena, M., et al. 2007, ApJS, 172, 132 Bisbas, T. G., Papadopoulos, P. P., \& Viti, S. 2015, ApJ, 803, 37 Bisbas, T. G., van Dishoeck, E. F., Papadopoulos, P. P., et al. 2017, ApJ, 839, 90

Bolatto, A. D., Wolfire, M., \& Leroy, A. K. 2013, ARA\&A, 51, 207 Bothwell, M. S., Aguirre, J. E., Aravena, M., et al. 2017, MNRAS, 466, 2825 Bothwell, M. S., Smail, I., Chapman, S. C., et al. 2013, MNRAS, 429, 3047 Bournaud, F., Daddi, E., Weiß, A., et al. 2015, A\&A, 575, A56

Bourne, N., Dunlop, J. S., Simpson, J. M., et al. 2018, MNRAS, in press (arXiv:1810.01640)

Bruzual, G., \& Charlot, S. 2003, MNRAS, 344, 1000

Carilli, C. L., Menten, K. M., \& Yun, M. S. 1999, ApJL, 521, L25

Carilli, C. L., \& Walter, F. 2013, ARA\&A, 51, 105

Casey, C. M., Berta, S., Béthermin, M., et al. 2012, ApJ, 761, 140

Casey, C. M., Narayanan, D., \& Cooray, A. 2014, PhR, 541, 45

Catalano, A., Calvo, M., Ponthieu, N., et al. 2014, A\&A, 569, A9

Chabrier, G. 2003, PASP, 115, 763

Chiappini, C., Romano, D., \& Matteucci, F. 2003, MNRAS, 339, 63

Cortzen, I., Garrett, J., Magdis, G., et al. 2019, MNRAS, 482, 1618

Cox, P., Krips, M., Neri, R., et al. 2011, ApJ, 740, 63

Daddi, E., Bournaud, F., Walter, F., et al. 2010a, ApJ, 713, 686

Daddi, E., Dannerbauer, H., Liu, D., et al. 2015, A\&A, 577, A46

Daddi, E., Dickinson, M., Morrison, G., et al. 2007, ApJ, 670, 156

Daddi, E., Elbaz, D., Walter, F., et al. 2010b, ApJL, 714, L118

Draine, B. T., \& Li, A. 2007, ApJ, 657, 810

Efstathiou, A., \& Siebenmorgen, R. 2009, A\&A, 502, 541

Elbaz, D., Daddi, E., Le Borgne, D., et al. 2007, A\&A, 468, 33

Emonts, B. H. C., Lehnert, M. D., Dannerbauer, H., et al. 2018, MNRAS, 477, L60

Frerking, M. A., Keene, J., Blake, G. A., \& Phillips, T. G. 1989, ApJ, 344, 311 Geach, J. E., Dunlop, J. S., Halpern, M., et al. 2017, MNRAS, 465, 1789

Genzel, R., Tacconi, L. J., Gracia-Carpio, J., et al. 2010, MNRAS, 407, 2091

Genzel, R., Tacconi, L. J., Lutz, D., et al. 2015, ApJ, 800, 20

Gerin, M., \& Phillips, T. G. 2000, ApJ, 537, 644

Glover, S. C. O., Clark, P. C., Micic, M., \& Molina, F. 2015, MNRAS, 448,1607

Guilloteau, S., \& Lucas, R. 2000, in ASP Conf. Ser. 217, Imaging at Radio through Submillimeter Wavelengths, ed. J. G. Mangum \& S. J. E. Radford (San Francisco, CA: ASP), 299

Guilloteau, S., Omont, A., McMahon, R. G., Cox, P., \& Petitjean, P. 1997, A\&A, 328, L1

Gullberg, B., Lehnert, M. D., De Breuck, C., et al. 2016, A\&A, 591, A73

Ikeda, M., Oka, T., Tatematsu, K., Sekimoto, Y., \& Yamamoto, S. 2002, ApJS, 139,467

Israel, F. P., Rosenberg, M. J. F., \& van der Werf, P. 2015, A\&A, 578, A95 Ivison, R. J., Greve, T. R., Dunlop, J. S., et al. 2007, MNRAS, 380, 199 Ivison, R. J., Papadopoulos, P. P., Smail, I., et al. 2011, MNRAS, 412, 1913 Ivison, R. J., Swinbank, A. M., Swinyard, B., et al. 2010, A\&A, 518, L35 Jiao, Q., Zhao, Y., Zhu, M., et al. 2017, ApJL, 840, L18

Jin, S., Daddi, E., Liu, D., et al. 2018, ApJ, 864, 56

Juneau, S., Dickinson, M., Alexander, D. M., \& Salim, S. 2011, ApJ, 736, 104

Kamenetzky, J., Rangwala, N., \& Glenn, J. 2017, MNRAS, 471, 2917
Kamenetzky, J., Rangwala, N., Glenn, J., Maloney, P. R., \& Conley, A. 2016, ApJ, 829, 93

Kaplan, E. L., \& Meier, P. 1958, J. Am. Stat. Assoc., 53, 282

Kaufman, M. J., Wolfire, M. G., Hollenbach, D. J., \& Luhman, M. L. 1999, ApJ, 527, 795

Keene, J., Lis, D. C., Phillips, T. G., \& Schilke, P. 1996, in IAU Symp.178, Molecules in Astrophysics: Probes Processes, ed. E. F. van Dishoeck (Cambridge: Cambridge Univ. Press), 129

Kelly, B. C. 2007, ApJ, 665, 1489

Kennicutt, R. C., Jr. 1998a, ARA\&A, 36, 189

Kennicutt, R. C., Jr. 1998b, ApJ, 498, 541

Kirkpatrick, A., Pope, A., Alexander, D. M., et al. 2012, ApJ, 759, 139

Kovács, A., Chapman, S. C., Dowell, C. D., et al. 2006, ApJ, 650, 592

Kroupa, P. 2001, MNRAS, 322, 231

Laigle, C., McCracken, H. J., Ilbert, O., et al. 2016, ApJS, 224, 24

Lestrade, J.-F., Combes, F., Salomé, P., et al. 2010, A\&A, 522, L4

Liu, D., Daddi, E., Dickinson, M., et al. 2018, ApJ, 853, 172

Liu, D., Gao, Y., Isaak, K., et al. 2015, ApJL, 810, L14

Lu, N., Zhao, Y., Díaz-Santos, T., et al. 2017, ApJS, 230, 1

Lutz, D., Poglitsch, A., Altieri, B., et al. 2011, A\&A, 532, A90

Ma, J., Gonzalez, A. H., Spilker, J. S., et al. 2015, ApJ, 812, 88

Magdis, G. E., Daddi, E., Béthermin, M., et al. 2012a, ApJ, 760, 6

Magdis, G. E., Daddi, E., Béthermin, M., et al. 2012b, ApJ, 760, 6

Magdis, G. E., Elbaz, D., Hwang, H. S., et al. 2010, MNRAS, 409, 22

Magdis, G. E., Rigopoulou, D., Daddi, E., et al. 2017, A\&A, 603, A93

Magnelli, B., Lutz, D., Santini, P., et al. 2012, A\&A, 539, A155

Mannucci, F., Cresci, G., Maiolino, R., Marconi, A., \& Gnerucci, A. 2010, MNRAS, 408, 2115

Maraston, C. 2005, MNRAS, 362, 799

Markwardt, C. B. 2009, in ASP Conf. Ser. 411, Astronomical Data Analysis Software and Systems XVIII, ed. D. A. Bohlender, D. Durand, \& P. Dowler (San Francisco, CA: ASP), 251

McKean, J. P., Berciano Alba, A., Volino, F., et al. 2011, MNRAS, 414, L11

McMullin, J. P., Waters, B., Schiebel, D., Young, W., \& Golap, K. 2007, in ASP Conf. Ser. 376, Astronomical Data Analysis Software and Systems XVI, ed. R. A. Shaw, F. Hill, \& D. J. Bell (San Francisco, CA: ASP), 127

Menéndez-Delmestre, K., Blain, A. W., Smail, I., et al. 2009, ApJ, 699, 667

Mullaney, J. R., Alexander, D. M., Goulding, A. D., \& Hickox, R. C. 2011, MNRAS, 414, 1082

Muzzin, A., Wilson, G., Demarco, R., et al. 2013, ApJ, 767, 39

Noeske, K. G., Weiner, B. J., Faber, S. M., et al. 2007, ApJL, 660, L43

Ojha, R., Stark, A. A., Hsieh, H. H., et al. 2001, ApJ, 548, 253

Oliver, S. J., Bock, J., Altieri, B., et al. 2012, MNRAS, 424, 1614

Oteo, I., Ivison, R. J., Dunne, L., et al. 2018, ApJ, 856, 72

Ott, S. 2010, in ASP Conf. Ser. 434, Astronomical Data Analysis Software and Systems XIX, ed. Y. Mizumoto, K.-I. Morita, \& M. Ohishi (San Francisco, CA: ASP), 139

Papadopoulos, P. P., Bisbas, T. G., \& Zhang, Z. Y. 2018, MNRAS, 478, 1716

Papadopoulos, P. P., \& Greve, T. R. 2004, ApJL, 615, L29

Papadopoulos, P. P., Thi, W.-F., \& Viti, S. 2004, MNRAS, 351, 147

Papovich, C., Labbé, I., Glazebrook, K., et al. 2016, NatAs, 1, 0003

Popping, G., Decarli, R., Man, A. W. S., et al. 2017, A\&A, 602, A11

Sanders, D. B., Mazzarella, J. M., Kim, D.-C., Surace, J. A., \& Soifer, B. T. 2003, AJ, 126, 1607

Sanders, D. B., Salvato, M., Aussel, H., et al. 2007, ApJS, 172, 86

Sargent, M. T., Daddi, E., Béthermin, M., et al. 2014, ApJ, 793, 19

Schmidt, M. 1959, ApJ, 129, 243

Scoville, N., Aussel, H., Brusa, M., et al. 2007, ApJS, 172, 1

Scoville, N., Aussel, H., Sheth, K., et al. 2014, ApJ, 783, 84

Skrutskie, M. F., Cutri, R. M., Stiening, R., et al. 2006, AJ, 131, 1163

Solomon, P. M., \& Vanden Bout, P. A. 2005, ARA\&A, 43, 677

Stacey, H. R., McKean, J. P., Robertson, N. C., et al. 2018, MNRAS, 476,5075

Stoerzer, H., Stutzki, J., \& Sternberg, A. 1997, A\&A, 323, L13

Stutzki, J., Bensch, F., Heithausen, A., Ossenkopf, V., \& Zielinsky, M. 1998 A\&A, 336, 697

Stutzki, J., Graf, U. U., Haas, S., et al. 1997, ApJL, 477, L33

Swinbank, A. M., Smail, I., Longmore, S., et al. 2010, Natur, 464, 733

Tacconi, L. J., Genzel, R., Saintonge, A., et al. 2018, ApJ, 853, 179

Tacconi, L. J., Neri, R., Chapman, S. C., et al. 2006, ApJ, 640, 228

U, V., Sanders, D. B., Mazzarella, J. M., et al. 2012, ApJS, 203, 9

van Dishoeck, E. F., \& Black, J. H. 1988, ApJ, 334, 771

Véron-Cetty, M.-P., \& Véron, P. 2010, A\&A, 518, A10

Vieira, J. D., Crawford, T. M., Switzer, E. R., et al. 2010, ApJ, 719, 763 
Wagg, J., Carilli, C. L., Aravena, M., et al. 2014, ApJ, 783, 71

Walter, F., Weiß, A., Downes, D., Decarli, R., \& Henkel, C. 2011, ApJ, 730, 18

Weiß, A., De Breuck, C., Marrone, D. P., et al. 2013, ApJ, 767, 88

Weiß, A., Downes, D., Henkel, C., \& Walter, F. 2005, A\&A, 429, L25
Weiß, A., Henkel, C., Downes, D., \& Walter, F. 2003, A\&A, 409, L41 Xie, T., Allen, M., \& Langer, W. D. 1995, ApJ, 440, 674

Yang, C., Omont, A., Beelen, A., et al. 2017, A\&A, 608, A144

Zanella, A., Daddi, E., Magdis, G., et al. 2018, MNRAS, 481, 1976 